\title{
The propagation of the shock wave from a strong explosion in a plane-parallel stratified medium: the Kompaneets approximation
}

\author{
C. A. Olano ${ }^{\star}$ \\ Universidad Nacional de La Plata, Facultad de Ciencias Astronómicas y Geofísicas, Paseo del Bosque, 1900 La Plata, Argentina \\ e-mail: colano@fcaglp.fcaglp.unlp.edu.ar
}

Received 29 May 2009 / Accepted 30 July 2009

\begin{abstract}
Context. Using certain simplifications, Kompaneets derived a partial differential equation that states the local geometrical and kinematical conditions that each surface element of a shock wave, created by a point blast in a stratified gaseous medium, must satisfy. Kompaneets could solve his equation analytically for the case of a wave propagating in an exponentially stratified medium, obtaining the form of the shock front at progressive evolutionary stages. Complete analytical solutions of the Kompaneets equation for shock wave motion in further plane-parallel stratified media were not found, except for radially stratified media.

Aims. We aim to analytically solve the Kompaneets equation for the motion of a shock wave in different plane-parallel stratified media that can reflect a wide variety of astrophysical contexts. We were particularly interested in solving the Kompaneets equation for a strong explosion in the interstellar medium of the Galactic disk, in which, due to intense winds and explosions of stars, gigantic gaseous structures known as superbubbles and supershells are formed.

Methods. Using the Kompaneets approximation, we derived a pair of equations that we call adapted Kompaneets equations, that govern the propagation of a shock wave in a stratified medium and that permit us to obtain solutions in parametric form. The solutions provided by the system of adapted Kompaneets equations are equivalent to those of the Kompaneets equation. We solved the adapted Kompaneets equations for shock wave propagation in a generic stratified medium by means of a power-series method.

Results. Using the series solution for a shock wave in a generic medium, we obtained the series solutions for four specific media whose respective density distributions in the direction perpendicular to the stratification plane are of an exponential, power-law type (one with exponent $k=-1$ and the other with $k=-2$ ) and a quadratic hyperbolic-secant. From these series solutions, we deduced exact solutions for the four media in terms of elemental functions. The exact solution for shock wave propagation in a medium of quadratic hyperbolic-secant density distribution is very appropriate to describe the growth of superbubbles in the Galactic disk.
\end{abstract}

Key words. hydrodynamics - shock waves - Galaxy: disk - ISM: bubbles - ISM: supernova remnants

\section{Introduction}

The study of the dynamics of a non-uniform gaseous medium under the effects of an intense point explosion or impact is of great astrophysical interest. The exact theoretical formulation of the problem leads to a coupled system of governing partial differential equations for the fluid dynamics, which is usually so complicated that it must be integrated numerically by means of refined computational techniques. Nevertheless, Kompaneets (1960) found an analytic solution for a blast wave propagating in an exponentially stratified ambient medium under certain simplifying assumptions. The comparison with accurate numerical solutions shows that the analytic approach of Kompaneets works surprisingly well (see review by Bisnovatyi-Kogan \& Silich 1995, and references therein). Alternative approaches to analyze the evolution of blast waves in a nonuniform medium confirm the Kompaneets results (Laumbach \& Probstein 1969; Koo \& McKee 1990).

The original motivation of the Kompaneets investigation was the description of the gas dynamics effects of a high energy

\footnotetext{
^ Member of the Carrera del Investigador Científico del CONICET, Argentina.
}

nuclear explosion in the Earth's upper atmosphere. In an astrophysical context, a similar phenomenon is the explosion of a supernova $(\mathrm{SN})$, which produces a strong shock wave in the interstellar medium (ISM). Various authors have investigated the evolution of SN remnants, using the Kompaneets solution (Gulliford 1974; Rosado 1981; Lozinskaya 1992; Maciejewski \& Cox 1999). The Kompaneets solution has been used and adapted for application to various astrophysical phenomena: multiple supernovae and stellar winds from $\mathrm{OB}$ associations (Icke 1988; Basu et al. 1999; Dove et al. 2000; Spitoni et al. 2008), relativistic blast waves (Shapiro1979), active galaxy winds (Schiano1985), and impacts within the deep gaseous envelopes of giant planets (Korycansky 1992) and onto the Earth's surface (Newman et al. 1999).

Bisnovatyi-Kogan \& Silich (1995) provide a comprehensive review of astrophysical shock waves in which they present the different numerical and analytical methods, including the Kompaneets (1960) approximation, to address the problem of shock wave propagation in the nonuniform ISM. From the Rankine-Hugoniot conditions for the velocity of a strong shock front and the assumptions that: (a) the pressure behind the shock front is spatially uniform and depends only on time, and (b) each surface element of the shock front moves perpendicular to 
itself, Kompaneets (1960) derived a partial differential equation whose solution gives the shape and evolution of a shock front created by a strong point explosion in a static stratified medium. Kompaneets solved his equation analytically for the particular case of a shock front propagating in a plane exponential atmosphere, i.e. with a specific law of vertical stratification. Thus, we will use the term "the Kompaneets solution" for the analytic expression of the shape of a shock front and, by extension, of the shell of gas swept up by the shock front during its expansion through an exponential atmosphere.

The Kompaneets equation by which Kompaneets obtained his particular solution could be used in principle to solve analytically the wave propagation in different media from the exponential atmosphere. So far, the Kompaneets equation has only been solved for an exponential medium, excepting solutions for radially stratified media with a power-law density distribution (Korycansky 1992) and an asymptotic solution for a planeparallel stratified medium with inverse-square decreasing density (Kontorovich \& Pimenov 1998). Hence, our objective is to apply the Kompaneets approximation to plane-parallel stratified atmospheres with other density distributions.

Our primary motivation is the study of large-scale expanding structures in the ISM, known as supershells and superbubbles. These objects are envisaged as holes or cavities in the Galactic gaseous disk and have sizes from 100 to over 1000 pc (Heiles 1979; Dickey \& Lockman 1990). The interstellar gas is concentrated strongly towards the Galactic plane. Hence, the gaseous disk of the Galaxy can be represented by stratified layers, parallel to the Galactic plane, whose densities decrease with the height above or below the Galactic plane. Since one of our aims is to obtain approximate formulae describing the evolution of supershells in the Galactic disk, we will apply the Kompaneets approximation to different stratified media that can represent the vertical density distribution of the Galactic disk (Dickey \& Lockman 1990).

In Sect. 2, we outline the Kompaneets model, which is linked to the adoption of an exponential atmosphere as the medium of shock-wave propagation. In Sect. 3, we extend the Kompaneets model to address the problem of shock-wave propagation in a generic stratified atmosphere. In Sects. 4 and 5, we demonstrate that the general solution, obtained in a power series for the generic atmosphere, leads to exact solutions in terms of elemental functions when applied to certain specific atmospheres: with exponential (Sect. 4.1), power-law-type (Sects. 4.2 and 4.3) and $\operatorname{sech}^{2}$ (Sect. 5) density distributions. Finally, in Sect. 6 we give a summary and conclusions of this work.

\section{The Kompaneets approximation}

The method we delineate in this section and use throughout the paper is based on that developed originally by Kompaneets (1960) and must not be confused with the thin-shell (or layer) approximation, which is sometimes called by the same name (see Bisnovatyi-Kogan \& Silich 1995, for a clear distinction between both methods).

We will study the evolution of a shock front referred to a cylindrical system of coordinate $(r, z)$, where $z$ is perpendicular to the stratification plane. In the Kompaneets model, the origin of the coordinate system is located at the explosion position (or energy point source). Since there is symmetry around the $z$-axis, we can omit the azimuthal angle. Therefore the evolution of a shock front generated by a point explosion can be represented by the function $f(r, z, t)=0$. The shock front surface in three dimensions at a given time $t$ is then obtained by rotating the closed curve $f(r, z, t)=0$ around the $z$-axis. Since $\frac{\mathrm{d} f}{\mathrm{~d} t}=0$, we get

$\frac{\partial f}{\partial r} \frac{\mathrm{d} r}{\mathrm{~d} t}+\frac{\partial f}{\partial z} \frac{\mathrm{d} z}{\mathrm{~d} t}+\frac{\partial f}{\partial t}=\boldsymbol{v} \cdot \nabla f+\frac{\partial f}{\partial t}=0$

where $\nabla f$ is the gradient of $f$ and $v$ is the velocity vector of the wave front, whose components are $\frac{\mathrm{d} r}{\mathrm{~d} t}$ and $\frac{\mathrm{d} z}{\mathrm{~d} t}$. In the Kompaneets approximation (Kompaneets 1960), it is assumed that the velocity vector of each surface element of the wave front is perpendicular to its own surface element. The function $f(r, z, t)=0$ at a given time $t$ is the level surface of $f$ representing the surface of the wave front at this time. Therefore, $\nabla f$ at any given position on the wave front is perpendicular to the surface at the corresponding position. Then, $\nabla f$ and $v$ are parallel vectors and $\boldsymbol{v} \cdot \nabla f=|\boldsymbol{v}| \cdot|\nabla f|$. Hence the modulus of $\boldsymbol{v}$ is (from Eq. (1))

$v=|\boldsymbol{v}|=-\frac{\partial f / \partial t}{|\nabla f|}$

We assume that the equation $f(r, z, t)=0$ is solved for $r$ as a function of $z$ and $t$, i.e. $r=g(z, t)$. Therefore $f(r, z, t)=r-$ $g(z, t)=0, \frac{\partial f}{\partial z}=-\frac{\partial g}{\partial z}=-\frac{\partial r}{\partial z}$ and $\frac{\partial f}{\partial r}=1$. We then have that

$|\nabla f|=\sqrt{\left(\frac{\partial f}{\partial r}\right)^{2}+\left(\frac{\partial f}{\partial z}\right)^{2}}=\sqrt{1+\left(\frac{\partial r}{\partial z}\right)^{2}}$.

With Eq. (3) and $\frac{\partial f}{\partial t}=-\frac{\partial g}{\partial t}=-\frac{\partial r}{\partial t}$, Eq. (2) becomes

$\left(\frac{\partial r}{\partial t}\right)^{2}-v^{2}\left[1+\left(\frac{\partial r}{\partial z}\right)^{2}\right]=0$.

Assuming that the internal pressure of the bubble, $P(t)$, dominates any external pressure, $v$ is given by the Hugoniot relations for a strong shock,

$v=\sqrt{\frac{\gamma+1}{2} \frac{P(t)}{\rho(z)}}$,

where $\gamma$ is the ratio of specific heats, and $\rho(z)=\rho_{0} F(z)$ is the initial density distribution of the ambient gas. Considering that the internal pressure is uniform, this can be expressed in terms of the energy density as

$P(t)=(\gamma-1) \lambda(\gamma) \frac{E}{V(t)}$,

where $E$ is the total energy of the explosion, $V(t)$ is the volume enclosed by the blast wave, and $\lambda(\gamma)$ is a numerical coefficient that can be estimated from the solution to the explosion problem in a homogeneous medium. Replacing Eq. (6) in Eq. (5), we obtain

$v^{2}=\frac{\lambda E\left(\gamma^{2}-1\right)}{2 \rho_{0} V(t)} F(z)^{-1}$.

In order to simplify the problem, Kompaneets (1960) incorporated an auxiliary variable $y$ defined by

$y=\int_{0}^{t} \sqrt{\frac{\lambda E\left(\gamma^{2}-1\right)}{2 \rho_{0}}} \frac{\mathrm{d} t}{\sqrt{V(t)}}$.

Substituting Eq. (7) and transforming the time variable according to Eq. (8), Eq. (4) reduces to:

$\left(\frac{\partial r}{\partial y}\right)^{2}-F(z)^{-1}\left[1+\left(\frac{\partial r}{\partial z}\right)^{2}\right]=0$. 
This is the fundamental equation obtained by Kompaneets (1960), who showed that Eq. (9) can be solved analytically by separation of variables for the case of an exponential atmosphere $F(z)=\exp (-z / H)$, yielding the solution

$r=g\left(z, t_{\star}\right)=2 H \arccos \left[\frac{1}{2} \mathrm{e}^{z / 2 H}\left(1-t_{\star}^{2}+\mathrm{e}^{-z / H}\right)\right]$,

where $t_{\star}=\frac{y}{2 H}$.

\section{Adapted Kompaneets' equations: a power-series method for solving them}

The solutions of Eq. (9), as Eq. (10), describe the shape of a shock front as a function of the time-like variable $t_{\star}$, but they say nothing about the trajectories of the parts that form the surface of the shock wave. It is useful to be able to determine the trajectories and velocities of individual particles associated with the shock front. The Kompaneets model describes the motion of the shock front, formally representing the shock discontinuity as a mathematical surface infinitesimally thin. Even though the dynamics of the rear disturbed gas layer that accompanies the shock front is not analyzed in the Kompaneets model, the trajectories of individual mathematical points of the shock front (streamlines) can give an approximate idea of the associated gasparticle trajectories. Indeed, a complete solution for the particular case of a strong point explosion in a homogeneous atmosphere, the Sedov (1959) solution, demonstrates that most of the swept-up shocked ambient gas is concentrated in a thin layer, just behind the shock front, and is moving at a velocity close to the velocity of the shock front (for more details see Zel'dovich \& Raizer 1968; Bisnovatyi-Kogan \& Silich 1995).

The trajectory or the so-called stream-line of each surface element of the wave front can be described by solutions in parametric form with $r\left(\varphi, t_{\star}\right)$ and $z\left(\varphi, t_{\star}\right)$, where $\varphi$ is the angle between the initial direction in which the surface element moves from the explosion point (i.e. at $t_{\star}=0$ ) and the stratification plane. We will call $\varphi$ the angle of departure, which determines the trajectory of the corresponding surface element. The point explosion produces a supersonically expanding sphere of hot gas, which acting as a piston, initially induces the formation of a concentric spherical shock front that runs ahead into the undisturbed surrounding gas. In other words, it is assumed that at times close to $t_{\star}=0$ the wave front is spherical and expands radially with uniform velocity. Hence $0 \leq \varphi \leq 2 \pi$.

To obtain solutions in parametric form, we will adapt the equations of the Kompaneets model outlined in Sect. 2. We express the solutions in terms of the variables $\varphi$ and $t_{\star}$. We define $t_{\star}=\frac{y}{2 H}$, where $y$ is is given by Eq. (8) and $H$ is the scale height of the density distribution $F(z)$. Hence $t_{\star}$ is a-dimensional. The velocity components of a point of the shock front are now expressed by $\frac{\mathrm{d} r}{\mathrm{~d} t}=\frac{\partial r}{\partial t_{\star}} \frac{\mathrm{d} t_{\star}}{\mathrm{d} t}$ and $\frac{\mathrm{d} z}{\mathrm{~d} t}=\frac{\partial z}{\partial t_{\star}} \frac{\mathrm{d} t_{\star}}{\mathrm{d} t}$. According to Eq. (8) and the definition of $t_{\star}, \frac{\mathrm{d} t_{\star}}{\mathrm{d} t}=\frac{1}{2 H \sqrt{V(t)}} \sqrt{\frac{\lambda E\left(\gamma^{2}-1\right)}{2 \rho_{0}}}$. Therefore $v^{2}=\left(\frac{\mathrm{d} r}{\mathrm{~d} t}\right)^{2}+\left(\frac{\mathrm{d} z}{\mathrm{~d} t}\right)^{2}=\left[\left(\frac{\partial r}{\partial t_{\star}}\right)^{2}+\left(\frac{\partial z}{\partial t_{\star}}\right)^{2}\right] \frac{1}{4 H^{2}} \frac{\lambda E\left(\gamma^{2}-1\right)}{2 \rho_{0} V(t)}$, and equating to Eq. (7), we get

$$
\left[\left(\frac{\partial r}{\partial t_{\star}}\right)^{2}+\left(\frac{\partial z}{\partial t_{\star}}\right)^{2}\right]-4 H^{2} F(z)^{-1}=0 .
$$

Equation (11) determines the modulus of $\boldsymbol{v}$, but we need a second condition to determine the direction of $\boldsymbol{v}$. The Kompaneets model demands that the velocity $v$ of a point of the shock front should be perpendicular to the part of the front formed by this point and its neighbors. For example, for a given $t_{\star}=t_{1}=$ const., the points $\left\{r\left(\varphi, t_{1}\right), z\left(\varphi, t_{1}\right)\right\}$ with $0 \leq \varphi \leq 2 \pi$ represent the surface of the wave front at this particular $t_{\star}$. Let $S_{1}$ be an arbitrary point on this surface. This point of coordinates $\left(r\left(\varphi_{1}, t_{1}\right), z\left(\varphi_{1}, t_{1}\right)\right)$ lies at the intersection of the trajectory of the point with angle $\varphi_{1}$ of departure and the wave surface at $t_{1}$. The coordinates of a point $S\left(r\left(\varphi_{1}+\mathrm{d} \varphi, t_{1}\right), z\left(\varphi_{1}+\mathrm{d} \varphi, t_{1}\right)\right)$ on the shock front close to $S_{1}$ can be expressed by $r\left(\varphi_{1}+\mathrm{d} \varphi, t_{1}\right)=r\left(\varphi_{1}, t_{1}\right)+\frac{\partial r}{\partial \varphi} \mathrm{d} \varphi$ and $z\left(\varphi_{1}+\mathrm{d} \varphi, t_{1}\right)=z\left(\varphi_{1}, t_{1}\right)+\frac{\partial z}{\partial \varphi} \mathrm{d} \varphi$, where the partial derivatives are evaluated at $\varphi=\varphi_{1}$ and $t_{\star}=t_{1}$. The vector from $S_{1}$ to $S$, which we call $\mathrm{d} S_{1}$, is a tangent vector to the wave surface at $S_{1}$. Since the vector between two points is the difference of the position vectors to the two points, $\mathrm{d} \boldsymbol{S}_{\mathbf{1}}=\boldsymbol{S}\left(r\left(\varphi_{1}, t_{1}\right)+\frac{\partial r}{\partial \varphi} \mathrm{d} \varphi, z\left(\varphi_{1}, t_{1}\right)+\right.$ $\left.\frac{\partial z}{\partial \varphi} \mathrm{d} \varphi\right)-S_{\mathbf{1}}\left(r\left(\varphi_{1}, t_{1}\right), z\left(\varphi_{1}, t_{1}\right)\right)=\left(\frac{\partial r}{\partial \varphi}, \frac{\partial z}{\partial \varphi}\right) \mathrm{d} \varphi$. Since the velocity $\boldsymbol{v}$ of the point $S_{1}$ should be perpendicular to the local surface of the shock wave, the scalar product of $\mathrm{d} \boldsymbol{S}_{\mathbf{1}}=\left(\frac{\partial r}{\partial \varphi}, \frac{\partial z}{\partial \varphi}\right) \mathrm{d} \varphi$ by $\boldsymbol{v}=\left(\frac{\partial r}{\partial t_{\star}}, \frac{\partial z}{\partial t_{\star}}\right) \frac{\mathrm{d} t_{\star}}{\mathrm{d} t}$ must be $\mathrm{d} \boldsymbol{S}_{\mathbf{1}} \cdot \boldsymbol{v}=0$. Therefore, the orthogonality relation at each point of the shock front is

$\frac{\partial r}{\partial \varphi} \frac{\partial r}{\partial t_{\star}}+\frac{\partial z}{\partial \varphi} \frac{\partial z}{\partial t_{\star}}=0$.

The simultaneous solution of Eqs. (11) and (12), which we will call the adapted Kompaneets equations, provides $r\left(\varphi, t_{\star}\right)$ and $z\left(\varphi, t_{\star}\right)$ that in the framework of the Kompaneets model describes in complete form the evolution of a shock front in a stratified medium. We suppose a series solution of the form

$$
\begin{aligned}
& r=r\left(\varphi, t_{\star}\right)=\sum_{n=1}^{\infty} r_{n}(\varphi) t_{\star}^{n} \\
& z=z\left(\varphi, t_{\star}\right)=\sum_{n=1}^{\infty} z_{n}(\varphi) t_{\star}^{n},
\end{aligned}
$$

where $r_{n}$ and $z_{n}$ are functions of $\varphi$ which remain to be determined. To solve Eqs. (11) and (12) using infinite series, we should also expand the term $F(z)^{-1}$ of Eq. (11) in a series of powers of $z$, which can generically be written as

$F(z)^{-1}=1+f_{1}\left(\frac{z}{H}\right)+f_{2}\left(\frac{z}{H}\right)^{2}+f_{3}\left(\frac{z}{H}\right)^{3}+\ldots$

Given $F(z)$, the numerical values of the coefficients $f_{n}$ are naturally obtained from $f_{n}=\frac{H^{n}}{n !} \frac{\mathrm{d}^{n}\left[F(0)^{-1}\right]}{\mathrm{d}^{n}}$; expressed in this way the coefficients $\mathrm{f}_{\mathrm{n}}$ are adimensional. We should substitute Eq. (14) in Eq. (15) and the result of the substitution in Eq. (11). We should also substitute $\frac{\partial r}{\partial t_{\star}}=\sum_{n=1}^{\infty} n r_{n} t_{\star}^{n-1}, \frac{\partial r}{\partial \varphi}=\sum_{n=1}^{\infty} \dot{r}_{n} t_{\star}^{n}$ and the similar expressions for $z$, derived from Eqs. (13) and (14), into Eqs. (11) and (12). After substituting, multiplying the series and summing the coefficients of the same power of $t_{\star}$, Eqs. (11) and (12) can be written as $\sum_{n=0}^{\infty} c_{n} t_{\star}^{n}=0$ and $\sum_{n=1}^{\infty} d_{n} t_{\star}^{n}=0$, respectively. This implies that

$c_{0}=c_{0}\left(r_{1}, z_{1}\right)=0$
$c_{1}=c_{1}\left(r_{1}, z_{1}, r_{2}, z_{2}\right)=0$

$c_{n}=c_{n}\left(r_{1}, z_{1}, \ldots, r_{n+1}, z_{n+1}\right)=0$, 
Table 1a. Coefficients $a_{r}(i, j)$ and $b_{r}(i, j)$ of the power series for $r$, corresponding to a stratified medium with a generic density distribution $F(z)^{a}$.

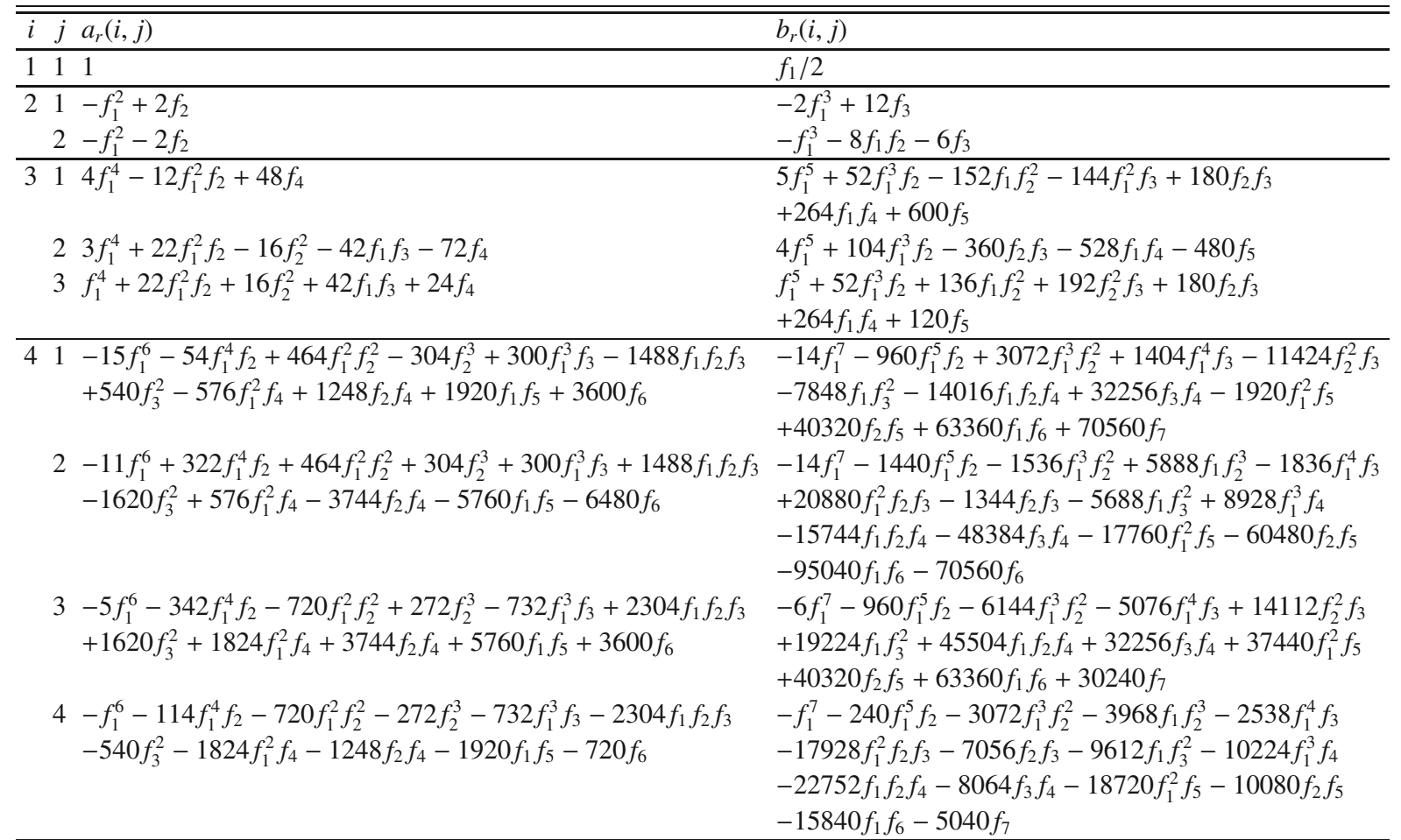

${ }^{a}$ The terms $f_{1}, f_{2}, f_{3}, \ldots$ represent the coefficients of the Taylor expansion of $F(z)^{-1}$, whose numerical values are determined from the specific distribution function $F(z)$ that we choose.

and

$d_{1}=d_{1}\left(r_{1}, \dot{r}_{1}, z_{1}, \dot{z}_{1}\right)=0$

$d_{2}=d_{2}\left(r_{1}, \dot{r}_{1}, z_{1}, \dot{z}_{1}, r_{2}, \dot{r}_{2}, z_{2}, \dot{z}_{2}\right)=0$

$d_{n}=d_{n}\left(r_{1}, \dot{r}_{1}, z_{1}, \dot{z}_{1}, \ldots, r_{n+1}, \dot{r}_{n+1}, z_{n+1}, \dot{z}_{n+1}\right)=0$

These relations allow us to determine $\left(r_{2}, z_{2}\right),\left(r_{3}, z_{3}\right),\left(r_{4}, z_{4}\right)$ and so on as a function of $\left(r_{1}, z_{1}\right)$, which is obtained from the initial conditions. Since the wave must be spherical for small $t_{\star}$, and the condition $c_{0}=r_{1}^{2}+z_{1}^{2}-4 H^{2}=0$ must be satisfied, we thus have $r_{1}=2 H \cos \varphi$ and $z_{1}=2 H \sin \varphi$. Solving recursively the rest of the coefficients (see Appendix A), we find the following solution of the system of equations (Eqs. (11) and (12))

$$
\begin{aligned}
r= & 2 H\left\{[\cos \varphi] t_{\star}+\frac{1}{2}\left[f_{1} \sin 2 \varphi\right] t_{\star}^{2}+\frac{1}{6}\left[-\left(f_{1}^{2}-2 f_{2}\right) \cos \varphi\right.\right. \\
& \left.-\left(f_{1}^{2}+2 f_{2}\right) \cos 3 \varphi\right] t_{\star}^{3}+\frac{1}{24}\left[-2\left(f_{1}^{3}-6 f_{3}\right) \sin 2 \varphi\right. \\
& \left.\left.-\left(f_{1}^{3}+8 f_{1} f_{2}+6 f_{3}\right) \sin 4 \varphi\right] t_{\star}^{4}+\ldots\right\} \\
z= & 2 H\left\{[\sin \varphi] t_{\star}-\frac{1}{2}\left[f_{1} \cos 2 \varphi\right] t_{\star}^{2}+\frac{1}{6}\left[-\left(f_{1}^{2}-2 f_{2}\right) \sin \varphi\right.\right. \\
& \left.-\left(f_{1}^{2}+2 f_{2}\right) \sin 3 \varphi\right] t_{\star}^{3}+\frac{1}{24}\left[\left(f_{1}^{3}-4 f_{1} f_{2}+6 f_{3}\right)+2\left(f_{1}^{3}\right.\right. \\
& \left.\left.\left.-6 f_{3}\right) \cos 2 \varphi+\left(f_{1}^{3}+8 f_{1} \mathrm{f}_{2}+6 f_{3}\right) \cos 4 \varphi\right] t_{\star}^{4}+\ldots\right\} .
\end{aligned}
$$

We see that the solutions for $r_{n}$ and $z_{n}$ are trigonometric polynomials. The coefficients $r_{n}$ with odd $n$ are finite linear combinations of $\cos (k \varphi)$ with $k=1,3,5, \ldots, n$. The solutions for $z_{n}$ with odd $n$ are similar to those of $r_{n}$, except the functions are $\sin (k \varphi)$. The coefficients of even powers with respect to $t_{\star}$ also have forms similar to those of odd powers just described above, with $\sin (k \varphi)$ and $\cos (k \varphi)$ interchanged, with $k=0,2,4, \ldots, n$. Equations (16) and (17) can be written in a more general form as follows:

$$
\begin{aligned}
r= & 2 H \sum_{i=1}^{\infty}\left\{\frac{1}{(2 i-1) !}\left[\sum_{j=1}^{i} a_{r}(i, j) \cos (2 j-1) \varphi\right] t_{\star}^{2 i-1}\right. \\
& \left.+\frac{1}{(2 i) !}\left[\sum_{j=1}^{i} b_{r}(i, j) \sin 2 j \varphi\right] t_{\star}^{2 i}\right\} . \\
z= & 2 H \sum_{i=1}^{\infty}\left\{\frac{1}{(2 i-1) !}\left[\sum_{j=1}^{i} a_{z}(i, j) \sin (2 j-1) \varphi\right] t_{\star}^{2 i-1}\right. \\
& \left.+\frac{1}{(2 i) !}\left[\sum_{j=1}^{i+1} b_{z}(i, j) \cos 2(j-1) \varphi\right] t_{\star}^{2 i}\right\} .
\end{aligned}
$$

A list of the coefficients $a_{r}(i, j), b_{r}(i, j), a_{z}(i, j)$ and $b_{z}(i, j)$ up to the terms of 8th order with respect to $t_{\star}$ is given in Tables $1 \mathrm{a}$ and $1 \mathrm{~b}$.

\section{Exact analytical solutions of the adapted Kompaneets equations for the shock-wave propagation in the exponential and other specific atmospheres}

We found exact analytical solutions of the adapted Kompaneets equations for media stratified with an exponential density 
Table 1b. Coefficients $a_{z}(i, j)$ and $b_{z}(i, j)$ of the power series for $z_{\star}$, corresponding to a stratified medium with an arbritrary density distribution.

\begin{tabular}{llll}
\hline \hline$i$ & $j$ & $a_{z}(i, j)$ & $b_{z}(i, j)$ \\
\hline 1 & 1 & 1 & 0 \\
& 2 & - & $-b_{r}(1,1)$ \\
\hline 2 & 1 & $a_{r}(2,1)$ & $f_{1}^{3}-4 f_{1} f_{2}+6 f_{3}$ \\
& 2 & $a_{r}(2,2)$ & $-b_{r}(2,1)$ \\
& 3 & - & $-b_{r}(2,2)$ \\
\hline 3 & 1 & $2 f_{1}^{4}-16 f_{2}^{2}-12 f_{1} f_{3}+96 f_{4}$ & $-4 f_{1}^{5}+16 f_{1}^{3} f_{2}-120 f_{2} f_{3}+48 f_{1} f_{4}+480 f_{5}$ \\
& 2 & $a_{r}(3,2)$ & $-7 f_{1}^{5}-36 f_{1}^{3} f_{2}+120 f_{1} f_{2}^{2}+120 f_{1}^{2} f_{3}-60 f_{2} f_{3}-312 f_{1} f_{4}-840 f_{5}$ \\
3 & $a_{r}(3,3)$ & $-b_{r}(3,2)$ \\
& $4-$ & $-b_{r}(3,3)$ \\
\hline 4 & 1 & $-5 f_{1}^{6}-114 f_{1}^{4} f_{2}+528 f_{1}^{2} f_{2}^{2}-240 f_{2}^{3}+324 f_{1}^{3} f_{3}$ & $15 f_{1}^{7}+168 f_{1}^{5} f_{2}-1440 f_{1}^{3} f_{2}^{2}+2112 f_{1} f_{2}^{3}-666 f_{1}^{4} f_{3}$ \\
& $-1440 f_{1} f_{2} f_{3}-540 f_{3}^{2}-384 f_{1}^{2} f_{4}+96 f_{2} f_{4}$ & $+5112 f_{1}^{2} f_{2} f_{3}-5040 f_{2} f_{3}-4860 f_{1} f_{3}^{2}+1296 f_{1}^{3} f_{4}-7200 f_{1} f_{2} f_{4}$ \\
& $+4800 f_{1} f_{5}+10800 f_{6}$ & $-4032 f_{3} f_{4}+2880 f_{1}^{2} f_{5}+10080 f_{2} f_{5}+50400 f_{1} f_{6}+75600 f_{7}$ \\
& $2-9 f_{1}^{6}-342 f_{1}^{4} f_{2}+528 f_{1}^{2} f_{2}^{2}+240 f_{2}^{3}+324 f_{1}^{3} f_{3}$ & $26 f_{1}^{7}+864 f_{1}^{5} f_{2}-2880 f_{1}^{3} f_{2}^{2}-1332 f_{1}^{4} f_{3}+10080 f_{2}^{2} f_{3}+9720 f_{1} f_{3}^{2}$ \\
& $+1440 f_{1} f_{2} f_{3}-1260 f_{3}^{2}+384 f_{1}^{2} f_{4}$ & $+14400 f_{1} f_{2} f_{4}-16128 f_{3} f_{4}-5760 f_{1}^{2} f_{5}-40320 f_{2} f_{5}-109440 f_{1} f_{6}-131040 f_{7}$ \\
& $-3360 f_{2} f_{4}-6720 f_{1} f_{5}-7920 f_{6}$ & \\
3 & $a_{r}(4,3)$ & $16 f_{1}^{7}+1416 f_{1}^{5} f_{2}+1632 f_{1}^{3} f_{2}^{2}-6016 f_{1} f_{2}^{3}+1872 f_{1}^{4} f_{3}$ \\
& & $-21312 f_{1}^{2} f_{2} f_{3}+2016 f_{2} f_{3}+4752 f_{1} f_{3}^{2}-8640 f_{1}^{3} f_{4}$ \\
& & $+15552 f_{1} f_{2} f_{4}+44352 f_{3} f_{4}+21600 f_{1}^{2} f_{5}+60480 f_{2} f_{5}$ \\
& & $+106560 f_{1} f_{6}+80640 f_{7}$ \\
& & $-b_{r}(4,3)$ \\
5 & $a_{r}(4,4)$ & $-b_{r}(4,4)$ \\
\hline
\end{tabular}

distribution, and with power-law-type density distributions. These solutions are exact in the sense we should be able to reconstruct the solution functions from their power series. Although the stratification laws we consider here are certainly ideal, they can fit partly or approximately real environments.

The density distribution of a stratified medium is generally expressed as a function of the altitude, or $Z$-height, above the ground level or symmetry plane. On the other hand, the Kompaneets model is referred to a local coordinate system, whose origin is the explosion point. Therefore, we need to establish a relation between both systems of reference. We choose the $Z$-axis, with the origin in the plane of symmetry, and the $z$-axis, with the origin in the explosion point, lying along a common line that passes through the explosion point and is perpendicular to the plane of symmetry. Both positive axes point in the same sense, toward decreasing density. Thus, the relation between both variables is $Z=z+Z_{0}$, where $Z_{0}$ is the $Z$ coordinate of the explosion point. Now our problem is to find the density distribution with respect to $z$, knowing the density distribution with respect to $Z$ : $\rho(Z)=\rho_{\mathrm{c}} G(Z)$, where $\rho_{\mathrm{c}}$ is the density in the ground level or symmetry plane (i.e. the maximum density). According to the well-known formula for the change of variables in distribution functions, $\rho(z)=\rho_{\mathrm{c}} G[\psi(z)] \frac{\mathrm{d} \psi}{\mathrm{d} z}$, where in our particular case $Z=\psi(z)=z+Z_{0}$ and hence $\frac{\mathrm{d} \psi}{\mathrm{d} z}=1$. Remembering that $\rho(z)=\rho_{0} F(z)$, where $\rho_{0}$ is the density in the explosion site, we have that $\rho_{0}=\rho_{\mathrm{c}} G\left(Z_{0}\right)$ and that

$F(z)=\frac{G\left(z+Z_{0}\right)}{G\left(Z_{0}\right)}$

The density distributions we use in this Section have in common the property that the transformation of Eq. (20) does not change the form of these distribution functions, except the value of the scale heights. In the case of the exponential distribution, even the scale height is the same after the transformation.

\subsection{Media stratified with an exponential density distribution}

We will apply the general solution provided by Eqs. (18) and (19) to the particular case of an exponential atmosphere, where $F(z)=\exp \left(-\frac{z}{H}\right)$. The Taylor series expansion of $F(z)^{-1}$ gives $F(z)^{-1}=\sum_{n=0}^{\infty} \frac{1}{n !}\left(\frac{z}{H}\right)^{n}$, whose comparison with Eq. (15) indicates that $f_{n}=\frac{1}{n !}$. Inserting these values of $f_{n}$ in their respective terms of the coefficients $a_{r}(i, j), b_{r}(i, j), a_{z}(i, j)$ and $b_{z}(i, j)$ given in Tables $1 \mathrm{a}$ and $1 \mathrm{~b}$, we find that if $j \neq i$, $a_{r}(i, j)=b_{r}(i, j)=0$, and if $j=i, a_{r}(i, j)=(-1)^{i+1}(2 i-2) !$ and $b_{r}(i, j)=(-1)^{i+1}(2 i-1)$ !. Besides, $a_{z}(i, j)=a_{r}(i, j)$ for $j=i$, $b_{z}(i, j)=-b_{r}(i, j-1)$ for $j=i+1$, and the remaining coefficients are zero. With these values of $a_{r}, b_{r}, \ldots$, Eqs. (18) and (19) take the simple form

$$
\begin{aligned}
& r=2 H\left[t_{\star} \cos \varphi+\frac{1}{2} t_{\star}^{2} \sin 2 \varphi-\frac{1}{3} t_{\star}^{3} \cos 3 \varphi-\frac{1}{4} t_{\star}^{4} \sin 4 \varphi+\ldots\right] \\
& z=2 H\left[t_{\star} \sin \varphi-\frac{1}{2} t_{\star}^{2} \cos 2 \varphi-\frac{1}{3} t_{\star}^{3} \sin 3 \varphi+\frac{1}{4} t_{\star}^{4} \cos 4 \varphi+\ldots\right] .
\end{aligned}
$$

Analyzing the known formulas $\sum_{k=1}^{\infty} \frac{p^{k} \sin k x}{k}=\arctan \frac{p \sin x}{1-p \cos x}$ and $\sum_{k=1}^{\infty} \frac{p^{k} \cos k x}{k}=\frac{1}{2} \ln \left(1-2 p \cos x+p^{2}\right)$ for $0<x<2 \pi$ and $p^{2} \leq 1$ (Grandshteyn \& Ryzhik 2000), it is easily inferred that Eqs. (21) and (22) result from the power series expansion of the following functions:

$$
\begin{aligned}
& r=2 H \arctan \left(\frac{t_{\star} \cos \varphi}{1-t_{\star} \sin \varphi}\right) \\
& z=-2 H \ln \left(\sqrt{1-2 t_{\star} \sin \varphi+t_{\star}^{2}}\right) .
\end{aligned}
$$

Therefore, Eqs. (23) and (24) give the exact parametric solution for an exponential density distribution. Applying the trigonometric identity $\cos u=\frac{1}{\sqrt{1+\tan ^{2} u}}$ to Eq. (23), we get $\cos \left(\frac{r}{2 H}\right)=$ $\frac{1-t_{\star} \sin \varphi}{\sqrt{1-2 t_{\star} \sin \varphi+t_{\star}^{2}}}$, from which and with Eq. (24) we can eliminate 
$\sin \varphi$ and obtain the Kompaneets solution (Eq. (10)). Instead, if we eliminate $t_{\star}$ from Eqs. (23) and (24), we obtain an equation for the orbit of a point on the shock front as follows

$z=-2 H \ln \left[\cos \varphi \sec \left(\varphi-\frac{r}{2 H}\right)\right]$.

The shape of the orbit is determined by the initial launch angle $\varphi$ of the point of the shock front.

Putting $\varphi=\pi / 2$ and $\varphi=-\pi / 2$ in Eq. (24), we obtain the position of the top, $-2 H \ln \left(1-t_{\star}\right)$, and of the bottom of the wave, $-2 H \ln \left(1+t_{\star}\right)$, respectively. At $t_{\star}=1$, the top formally reaches infinity and the bottom $z=-1.39 H$. In the Kompaneets model it is assumed implicitly that the density distribution function $F(z)$ holds for $-1.39 H \leq z<\infty$. However, in real situations, we generally have more strict boundary conditions. An example is the Galactic disk, whose density distribution can be represented by the double exponential function $\exp -\frac{|Z|}{H}$. Hence, the Galactic plane, $Z=0$, divides the space in two subspaces, each with its own density distribution. If the explosion point is at the height $Z_{0}$ above the Galactic plane (i.e. in the upper semi-space, $Z>0$ ), the density distribution with respect to $Z$, with the origin at the Galactic plane, is $\rho_{\mathrm{c}} \exp (-Z / H)$, where $\rho_{\mathrm{c}}$ is the density on the Galactic plane. In order to obtain the density distribution with respect to $z$, as the Kompannets model requires, we should replace $Z=Z_{0}+z$ in $\rho_{\mathrm{c}} \exp (-Z / H)=\rho_{\mathrm{c}} \exp \left(-Z_{0} / H\right) \exp (-z / H)$, see Eq. (20). Since $\rho_{\mathrm{c}} \exp \left(-Z_{0} / H\right)=\rho_{0}$, the density at the explosion site, $F(z)$ does not depend on $Z_{0}$ for this particular distribution. We should however remember that if a part of the front moving toward increasing densities crosses the Galactic plane (i.e. when $z \leq-Z_{0}$ ), the posterior evolution of this part of the front cannot be studied with the Kompaneets model. A special case is that of $Z_{0}=0$ in which we can consider separately one half of a wave moving in the upper semi-space, toward decreasing densities, and the other half of the wave moving in the lower semi-space, also toward decreasing densities. Therefore in this case the evolution of the wave front is symmetric with respect to the Galactic plane.

In Fig. 1, we show the evolution of a shock wave propagating in an exponential atmosphere of infinite extension and of extension limited by a boundary plane below the explosion site (e.g. the Galactic plane). In the example of Fig. $1, Z_{0}=H$ and therefore the region between $z=-H$ and $-1.39 H$ should be excluded from analysis. If the boundary plane is beyond $z<-1.39 H$ (or $Z_{0}>1.39 \mathrm{H}$ ), this does not affect the conditions of the Kompaneets model, that is to say, this case is equivalent to that of an infinite atmosphere. In Fig. 2, we show the evolution of the shock wave generated by a strong explosion in the midplane of a double exponential disk. To draw the curves of Figs. 1 and 2, we used the parametric representation given by Eqs. (23) and (24). Certainly, for this purpose, we could also use Eqs. (10) and (25). The application of the solution (Eqs. (23) and (24)) to the case of Fig. 2 requires some care. Given the symmetry conditions, it is only necessary to calculate the positions $(r, z)$ of the points of the shock front in the first quadrant; the other positions of the wave are obtained by $(-r, z),(-r,-z)$ and $(r,-z)$. We should take into account that the points of low $\varphi$ cross the midplane. Equating Eq. (24) to zero, we find $\sin \varphi=t_{\star} / 2$, indicating that at certain $t_{\star}=t_{1}$, points of the shock front with $\varphi_{0} \leq \arcsin t_{1} / 2$ crossed the midplane. Hence, the first quadrant is defined by $\varphi_{0} \leq \varphi \leq \pi / 2$. We assume that the interchange of particles, associated with points of the shock front of low $\varphi$, between the upper and lower quadrants does not alter essentially the conditions of the Kompaneets model.

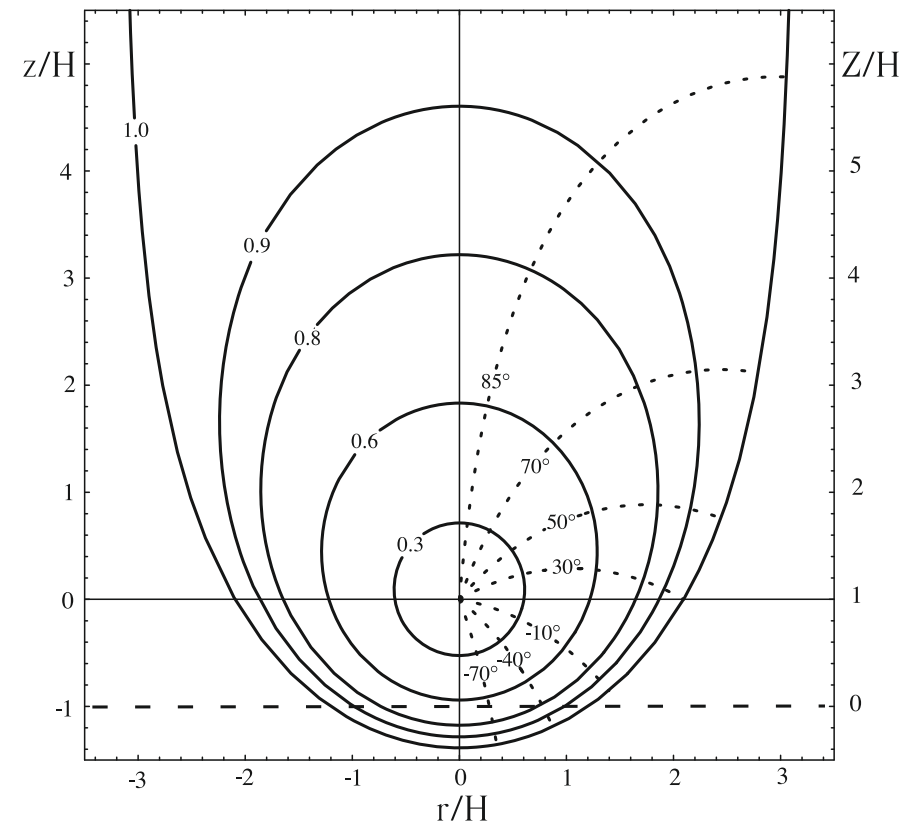

Fig. 1. Evolution of the shock front produced by a point explosion in a static exponential atmosphere. The solids curves show the shock front at successive stages of evolution, represented by the sequence of $t_{\star}$ : $\left\{t_{i}\right\}=\{0.3,0.6,0.8,0.9,1.0\}$. The curve for the shape of the shock front at $t_{i}$ is generated by setting $t_{\star}=t_{i}$ in Eqs. (23) and (24) and plotting $\left(r\left(\varphi, t_{i}\right), z\left(\varphi, t_{i}\right)\right)$ for $0 \leq \varphi \leq 2 \pi$. The dashed curves show trajectories or stream-lines of points of the front, launched from the explosion site with different angles: $\left\{\varphi_{i}\right\}=\left\{-70^{\circ},-40^{\circ},-10^{\circ}, 30^{\circ}, 50^{\circ}, 70^{\circ}, 85^{\circ}\right\}$. The curve for the trajectory of the point launched with the angle $\varphi_{i}$ is generated by setting $\varphi=\varphi_{i}$ in Eqs. (23) and (24) and plotting $\left(r\left(\varphi_{i}, t_{\star}\right), z\left(\varphi_{i}, t_{\star}\right)\right)$ for $0 \leq t_{\star} \leq 1$. To show an application to the case in which there is a boundary plane, such as the Galactic plane, this is indicated by a dashed line. The left hand vertical axis shows the coordinate $z$ referred to the explosion point. The right hand vertical axis shows the coordinate $Z$ referred to the Galactic plane. The correspondence between $t_{\star}$ and the elapsed time $t$ is given in Appendix B (see also Figs. B.1 and B.2).

\subsection{Media stratified with a power-law-type density distribution: $\frac{1}{1+\frac{Z}{H}}$}

We consider here an atmosphere with the density distribution $\rho(Z)=\frac{\rho_{\mathrm{c}}}{\left(1+\frac{Z}{H}\right)}$, where $Z$ is the height above the ground level (or the symmetry plane), $\rho_{\mathrm{c}}$ is the density at the ground level and $H$ the scale height. Applying Eq. (20), the density distribution, normalized to the density $\rho_{0}$ at the explosion point as a function of the height $z$ referred to the explosion point is $F(z)=\frac{1}{\left(1+\frac{z}{H_{\star}}\right)}$, where $H_{\star}=H+Z_{0}$, a new scale height, and $Z_{0}$ is the height $Z$ of the explosion point.

Comparing $F(z)^{-1}=1+\frac{z}{H_{\star}}$ with Eq. (15), we have $f_{1}=1$ and $f_{n}=0$ for $n \geq 2$. Substituting these coefficients in Tables $1 \mathrm{a}$ and $1 \mathrm{~b}$, we get the numerical values of $a_{r}(i, j), b_{r}(i, j), a_{z}(i, j)$ and $b_{z}(i, j)$ for this particular stratified medium, which are then inserted in Eqs. (18) and (19). To operate on Eq. (18), we can use the property $\cos (2 j-1) \varphi=T_{(2 j-1)}(\cos \varphi)$ and $\sin 2 j \varphi=$ $\sin \varphi U_{(2 j-1)}(\cos \varphi)$, where $T$ and $U$ are the Chebyshev polynomials of the first and second kind, respectively. We observe that the summation that represents the coefficient of the power $(2 i-1)$ of $t_{\star}$, or of the power $(2 i)$, becomes a polynomial of $\cos \varphi$ whose terms are canceled except the terms with the two major powers (i.e. $(2 i-1)$ and $(2 i-3))$, namely: $\frac{1}{2}\left[a_{r}(i, i)(2 \cos \varphi)^{2 i-1}+a_{r}(i, i-\right.$ 1) $\left.(2 \cos \varphi)^{2 i-3}-(2 i-1) a_{r}(i, i)(2 \cos \varphi)^{2 i-3}\right]$ for the odd powers 


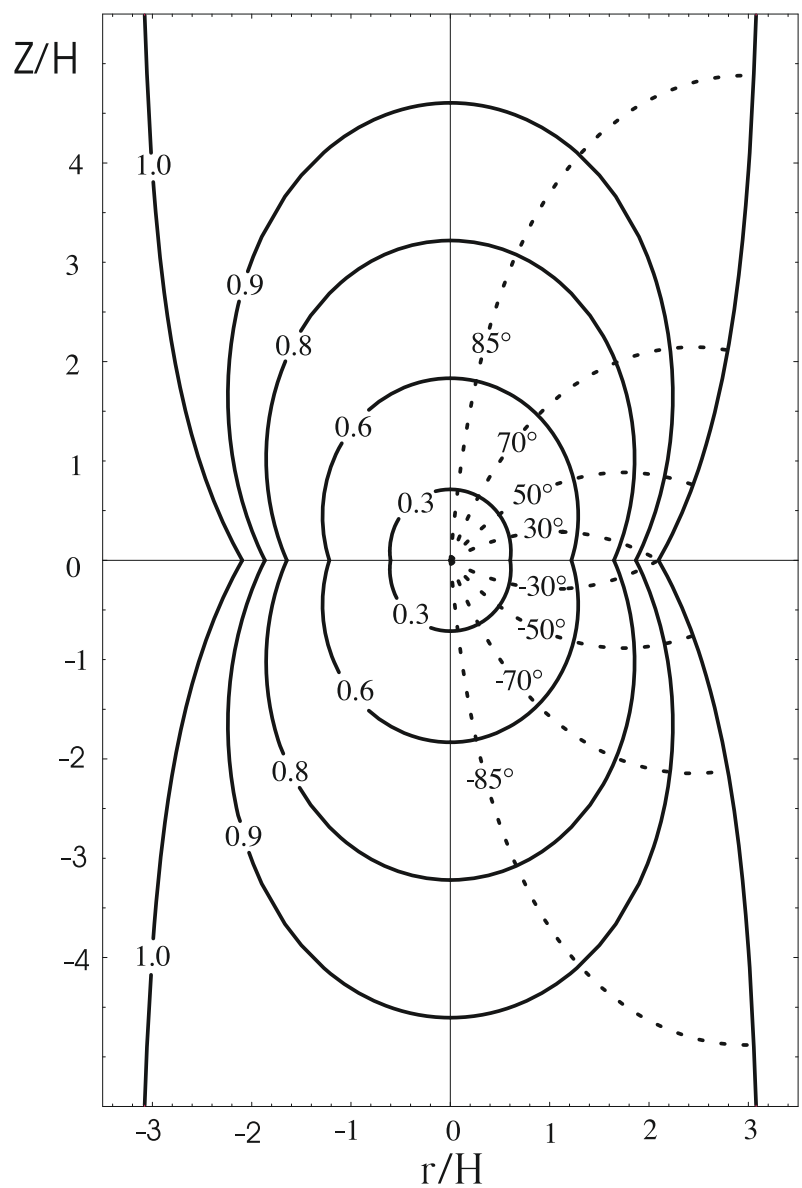

Fig. 2. Evolution of the shock front produced by a point explosion in the central plane $(Z=0)$ of a double exponential stratified medium. The burst point is located at the coordinate origin. Each solid curve shows the shape of the shock front at the value of the time-like parameter, $t_{\star}=t_{i}$, marked on it. Each dashed curve shows the path followed by the point on the shock front with the departure angle, $\varphi=\varphi_{i}$, marked on the curve. The correspondence between $t_{\star}$ and the elapsed time $t$ is given in Appendix B (see also Figs. B.1 and B.2).

of $t_{\star}$, and $\sin \varphi b_{r}(i, i)\left[(2 \cos \varphi)^{2 \mathrm{i}-1}-(2 i-2)(2 \cos \varphi)^{2 i-3}\right]+$ $\sin \varphi b_{r}(i, i-1)(2 \cos \varphi)^{2 i-3}$ for the even powers of $t_{\star}$. From Tables $1 \mathrm{a}$ and $1 \mathrm{~b}$, we obtained that $a_{r}(i, i)=(-1)^{i+1}$, $a_{r}(i, i-1)=(-1)^{i+1}(2 i-3), b_{r}(i, i)=(-1)^{i+1}$ and $b_{r}(i, i-1)=$ $(-1)^{i+1}(2 i-2)$. Therefore, Eq. (18) becomes

$$
\begin{aligned}
r= & 2 H_{\star}\left[t_{\star} \cos \varphi+\frac{\cos 2 \varphi}{(2 \cos \varphi)^{2}} \sum_{i=2}^{\infty} \frac{(-1)^{i+1}\left(2 t_{\star} \cos \varphi\right)^{2 i-1}}{(2 i-1) !}\right. \\
& \left.+\frac{\sin \varphi}{2 \cos \varphi} \sum_{i=1}^{\infty} \frac{(-1)^{i+1}\left(2 t_{\star} \cos \varphi\right)^{2 i}}{(2 i) !}\right] .
\end{aligned}
$$

The first and second summations of this equation are easily identified with $\sin \left(2 t_{\star} \cos \varphi\right)-2 t_{\star} \cos \varphi$ and with $1-\cos \left(2 t_{\star} \cos \varphi\right)$, respectively. Hence,

$$
\begin{aligned}
r= & 2 H_{\star}\left\{t_{\star} \cos \varphi+\frac{\cos 2 \varphi}{(2 \cos \varphi)^{2}}\left[\sin \left(2 t_{\star} \cos \varphi\right)-2 t_{\star} \cos \varphi\right)\right] \\
& \left.+\frac{\sin \varphi}{2 \cos \varphi}\left[1-\cos \left(2 t_{\star} \cos \varphi\right)\right]\right\} .
\end{aligned}
$$

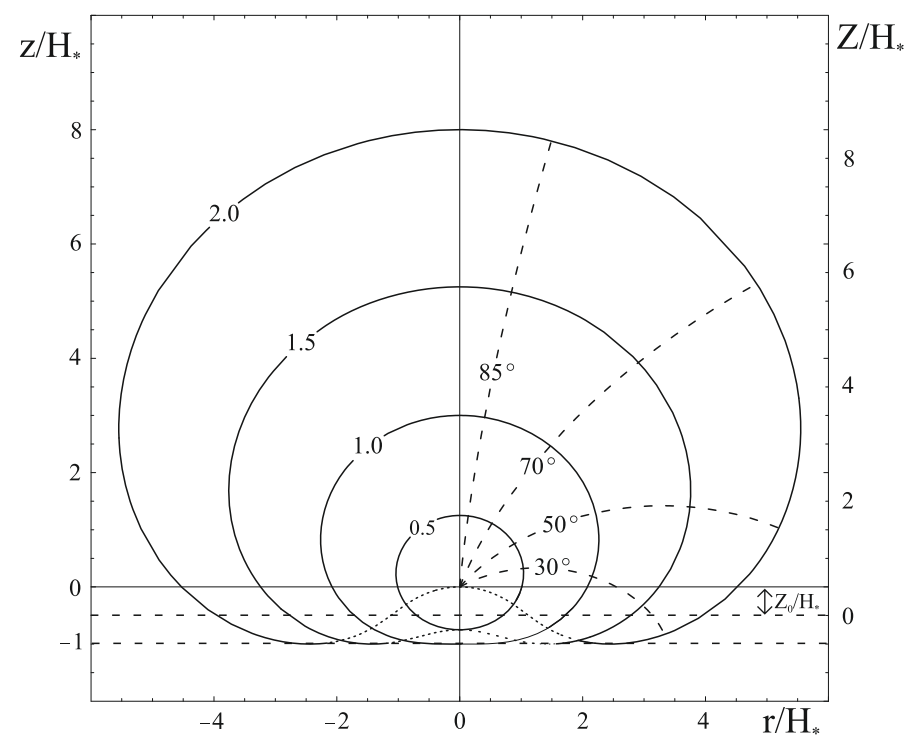

Fig. 3. Form of the shock wave (solid curves) at various evolutionary stages in a static gaseous medium with an initial density distribution given by the law: $\frac{1}{\left(1+\frac{Z}{H}\right)}$. See caption of Fig. 1 for the meaning of the different symbols. The correspondence between $t_{\star}$, whose represented values are marked on the solid curves, and the elapsed time is given in Appendix B.

Proceeding in a similar manner with Eq. (19), we find that this equation is reduced to

$$
\begin{aligned}
z= & 2 H_{\star}\left[\frac{\sin 2 \varphi}{2 \cos \varphi} \sum_{i=1}^{\infty} \frac{(-1)^{i+1}\left(2 t_{\star} \cos \varphi\right)^{2 i-1}}{(2 i-1) !}\right. \\
& \left.+\frac{\cos 2 \varphi}{(2 \cos \varphi)^{2}} \sum_{i=1}^{\infty} \frac{(-1)^{i+1}\left(2 t_{\star} \cos \varphi\right)^{2 i}}{(2 i) !}\right] .
\end{aligned}
$$

Note that the first summation is identical to the expan$\operatorname{sion}$ of $\sin \left(2 t_{\star} \cos \varphi\right)$, and the second summation is equal to $\cos \left(2 t_{\star} \cos \varphi\right)-1$. Hence, we are left with the final formula

$$
\begin{aligned}
z= & 2 H_{\star}\left\{\frac{\sin 2 \varphi}{2 \cos \varphi} \sin \left(2 t_{\star} \cos \varphi\right)\right. \\
& \left.+\frac{\cos 2 \varphi}{(2 \cos \varphi)^{2}}\left[\cos \left(2 t_{\star} \cos \varphi\right)-1\right]\right\} .
\end{aligned}
$$

Using Eqs. (26) and (27), we plot the curves of Fig. 3 representing the propagation of a shock wave in the gaseous medium investigated here. We see that the solid (and in parts dotted) curves for $t_{\star}>1$ look like cardioids. In fact, the curves of Fig. 3 can be approximately fitted by a function called the limaçon of Pascal, a generalization of the cardioid. In polar coordinates, this fitting function can be expressed by $\rho=a+b \sin \phi$, where in our case $a=2 H_{\star} t_{\star}, b=H_{\star} t_{\star}^{2}, \rho=\sqrt{r^{2}+z^{2}}$, and $\sin \phi=z / \sqrt{r^{2}+z^{2}}$. According to this limaçon of Pascal, the solution for the top of the wave, where $\phi=\pi / 2$, is $\rho=2 H_{\star} t_{\star}+H_{\star} t_{\star}^{2}$, which agrees with Eq. (27) when $\varphi \rightarrow \pi / 2$. Thus, the top of the wave is accelerated uniformly and does not blow out of the gaseous medium in a finite time, unlike the exponential atmosphere in which the top of the wave moves to infinity in a finite time $t_{b}=1$ (see Sect. 4.1). The plane $z / H_{\star}=-1$ has infinite density and hence cannot be overcame by the part of the wave that approaches this plane (see Fig. 3). Indeed, this part of the wave, which characterizes to the cardioid, is reflected inward (dotted line of Fig. 3). However, the Kompaneets model cannot describe properly the 
Table 2. Coefficients $a_{r}(i, j)$ and $b_{r}(i, j)$ of the power series for $r$, corresponding to a stratified medium with a $\frac{\rho_{\mathrm{C}}}{\left(1+\frac{Z}{H}\right)^{2}}$ law of density distribution in altitude.

\begin{tabular}{llll}
\hline \hline$i$ & $j$ & $a_{r}(i, j)$ & $b_{r}(i, j)$ \\
\hline 1 & 1 & 1 & 2 \\
\hline 2 & 1 & -2 & -16 \\
& 2 & -6 & -24 \\
\hline 3 & 1 & 16 & 272 \\
& 2 & 120 & 960 \\
& 3 & 120 & 720 \\
\hline
\end{tabular}

physical reality in this singular region, in part because the interior enclosed by the wave is almost empty. Besides, the ground level in our model is given by the plane $Z=z+Z_{0}=0$, which lies above the plane with infinite density. In other words, our analysis is valid only for $Z \geq 0$ or $z \geq-Z_{0}$. The particular case of an explosion in the symmetry plane of a disk with the double power-law-type density distribution $\frac{1}{1+\frac{L U}{H}}$ can be solved applying Eqs. (26) and (27) in a manner similar to that of the case of a double exponential disk (Sect. 4.1); and a display similar to Fig. 2 can be generated.

\subsection{Media stratified with a power-law-type density distribution: $\frac{1}{\left(1+\frac{Z}{H}\right)^{2}}$}

Here we consider an atmosphere with the density distribution $\rho(Z)=\frac{\rho_{\mathrm{c}}}{\left(1+\frac{Z}{H}\right)^{2}}$, where $Z$ is the height above the ground level (or the symmetry plane), $\rho_{\mathrm{c}}$ is the density at ground level and $H$ the scale height. Applying Eq. (20), the density distribution, normalized to the density $\rho_{0}$ at the explosion point, as a function of the height $z$ referred to the explosion point is $F(z)=\frac{1}{\left(1+\frac{z}{H_{\star}}\right)^{2}}$, where $H_{\star}=H+Z_{0}$, a new scale height, and $Z_{0}$ is the height $\mathrm{Z}$ of the explosion point. Hence $F(z)^{-1}=1+2 \frac{z}{H_{\star}}+\left(\frac{z}{H_{\star}}\right)^{2}$ and comparing with Eq. (15) we have that $f_{1}=2, f_{2}=1$, and $f_{n}=0$ for $n>2$. Replacing these values for the coefficients $f_{n}$ in Table 1 a and $1 \mathrm{~b}$, we obtain the values for $a_{r}, b_{r}, a_{z}$ and $b_{z}$. We find that $a_{z}(i, j)=a_{r}(i, j), b_{z}(i, 1)=0$, and $b_{z}(i, j)=-b_{r}(i, j-1)$ for $j>1$. Table 2 displays the values of the coefficients $a_{r}$ and $b_{r}$, up to $i=3$, with which we can also obtain the corresponding values of $a_{z}$ and $b_{z}$.

To gain some insight into the general solution, we first analyze the solution for the top of the wave: $z=H_{\star}\left(\mathrm{e}^{2 t_{\star}}-1\right)$, obtained by integrating Eq. (11), with $r=0$, by separation of variables. Using the identity $\mathrm{e}^{2 t_{\star}}-1=\frac{2}{\operatorname{coth} t_{\star}-1}$, we see that the general solution for $z$ can have the form:

$z=2 H_{\star} \frac{\sin \varphi-(\cos \varphi)^{2} u\left(t_{\star}\right)}{\operatorname{coth} t_{\star}-\sin \varphi+2(\cos \varphi)^{2} v\left(t_{\star}\right)}$,

since $\varphi=\pi / 2$ for the top of the wave. We assume that $u\left(t_{\star}\right)=$ $u_{1} t_{\star}+u_{3} t_{\star}^{3}+u_{5} t_{\star}^{5}+\ldots$ and $v\left(t_{\star}\right)=v_{1} t_{\star}+v_{3} t_{\star}^{3}+v_{5} t_{\star}^{5}+\ldots$ In order to obtain the values of $u_{1}, u_{3}, u_{5} \ldots$ and $v_{1}, v_{3}, v_{5} \ldots$, we compare the coefficients of equal power of the series expansion of Eq. (28) with those of the solution in a power series obtained substituting the coefficients of Table 2, and the rest of the coefficients derived from them, in Eq. (19). We take into account that the equivalent to $H$ in Eqs. (18) and (19) here is $H_{\star}$. The first terms of $z$ derived from Eq. (19) are $z=2 H_{\star}\left[t_{\star} \sin \varphi-t_{\star}^{2} \cos 2 \varphi-\frac{1}{3} t_{\star}^{3}(\sin \varphi+\right.$ $3 \sin 3 \varphi)+\ldots]$ and the first terms derived from the power-series expansion of Eq. (28) are $z=2 H_{\star}\left\{t_{\star} \sin \varphi+t_{\star}^{2}\left[-u_{1}(\cos \varphi)^{2}+\right.\right.$ $\left.\left.(\sin \varphi)^{2}\right]+\frac{1}{3} t_{\star}^{3} \sin \varphi\left[-1-3\left(u_{1}+2 v_{1}\right)(\cos \varphi)^{2}+3(\sin \varphi)^{2}\right]+\ldots\right\}$.
Equating the coefficients of $t_{\star}^{2}$ and of $t_{\star}^{3}$, we have two equations to find the two unknowns: $u_{1}$ and $v_{1}$. Similarly, equating the coefficients of $t_{\star}^{4}$ and of $t_{\star}^{5}$, we get the values of $u_{3}$ and $v_{3}$, and so on. The result is $u\left(t_{\star}\right)=v\left(t_{\star}\right)=t_{\star}+\frac{2}{3} t_{\star}^{3}+\frac{2}{15} t_{\star}^{5}+\frac{4}{315} t_{\star}^{7}+\ldots$, which is identical to the expansion of $\frac{1}{2} \sinh 2 t_{\star}$. Hence Eq. (28) becomes

$z=2 H_{\star} \frac{\sin \varphi-\frac{1}{2}(\cos \varphi)^{2} \sinh 2 t_{\star}}{\operatorname{coth} t_{\star}-\sin \varphi+(\cos \varphi)^{2} \sinh 2 t_{\star}}$.

In order to find the analytical solution for $r$, we see that a analytical form for the solution, compatible with the solution in power-series derived from Eq. (18), can be:

$r=2 H_{\star} \frac{t_{\star} \cos \varphi}{1-2 t_{\star} \sin \varphi+w\left(t_{\star}\right)}$,

where $w\left(t_{\star}\right)=w_{2} t_{\star}^{2}+w_{4} t_{\star}^{4}+w_{6} t_{\star}^{6}+\ldots$ Proceeding in a way similar to the example given in the previous paragraph, we get $w\left(t_{\star}\right)=\frac{4}{3} t_{\star}^{2}-\frac{16}{45} t_{\star}^{4}+\frac{128}{945} t_{\star}^{6}-\frac{256}{4725} t_{\star}^{8}+\ldots=-1+2 t_{\star} \operatorname{coth} 2 t_{\star}$. Then Eq. (30) takes the final form

$r=2 H_{\star} \frac{\cos \varphi}{2 \operatorname{coth} 2 t_{\star}-2 \sin \varphi}$

The general solutions for $r$ and $z$, Eqs. (31) and (29), satisfy the formula $r^{2}+\left(z-2 H_{\star} \sinh ^{2} t_{\star}\right)^{2}=\left(H_{\star} \sinh 2 t_{\star}\right)^{2}$. This means that the surface of the wave is a sphere with a center $z_{0}=2 H_{\star} \sinh ^{2} t_{\star}$ and a radius $\varrho=H_{\star} \sinh 2 t_{\star}$. In other words, the wave evolves as a spherical bubble that expands radially with the velocity $\frac{\mathrm{d} \varrho}{\mathrm{d} t_{\star}}$ and rises simultaneously with the velocity $\frac{\mathrm{d} z_{0}}{\mathrm{~d} t_{\star}}$. Now we rewrite the formula of the surface of the wave in canonical form as

$r=\sqrt{\left(H_{\star} \sinh 2 t_{\star}\right)^{2}-\left(z-2 H_{\star} \sinh ^{2} t_{\star}\right)^{2}}$,

with which we can easily verify that this equation fullfils the Kompaneets relation (Eq. (9)). Equations (31) and (29) also satisfy the following relation:

$\left(r-H_{\star} \tan \varphi\right)^{2}+\left(z+H_{\star}\right)^{2}=\left(H_{\star} \sec \varphi\right)^{2}$.

This shows that the orbits of the individual points are arcs of circles.

Figure 4 shows the evolution of the wave in the gaseous medium considered in this Section, according to Eqs. (29) and (31), or Eqs. (32) and (33). In the example of Fig. 4, the offset of the explosion point from the ground level or symmetry plane in the case of a disk is equal to $H_{\star} / 2$. The lower limit of the valid range of Eqs. (29) and (31) is the ground level or plane of symmetry. The explanation of this is similar to that given for the cases considered in Sects. 4.1 and 4.2. For the study of an explosion on the symmetry plane, such as for example the Galactic plane, we can employ Eqs. (29) and (31) following the procedure detailed in Sect. 4.1, by means of which we generated Fig. 2. The time that the wave takes to break out of this gaseous medium is formally infinity (i.e. $t_{b}=\infty$ ), as in the case discussed in the previous section.

\section{Exact analytical solution of the adapted Kompaneets equations for the shock-wave propagation in a medium stratified

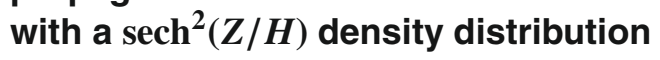

The density distribution we consider here is particularly appropriate to model the Z-distribution of the ISM. Assuming 
Table 3. Coefficients $a_{r}^{\star}(i, j)$ and $b_{r}^{\star}(i, j)$ of the power series for $r$, corresponding to a stratified medium with a sech ${ }^{2}(Z / H)$ density distribution.

\begin{tabular}{llll}
\hline \hline$i$ & $j$ & $a_{r}^{\star}(i, j)$ & $b_{r}^{\star}(i, j)$ \\
\hline 1 & 1 & 1 & 1 \\
\hline 2 & 1 & $-\frac{1}{3}(-1+\alpha)(1+\alpha)$ & $-\frac{2}{3}(-1+\alpha)(1+\alpha)$ \\
& 2 & $-\frac{1}{3}\left(1+3 \alpha^{2}\right)$ & $-1\left(1+\alpha^{2}\right)$ \\
\hline 3 & 1 & $\frac{2}{15}(-1+\alpha)^{2}(1+\alpha)^{2}$ & $\left.\frac{17}{45}(-1+\alpha)^{2}(1+\alpha)^{2}\right)$ \\
& 2 & $\frac{1}{3}(-1+\alpha)(1+\alpha)\left(1+3 \alpha^{2}\right)$ & $\frac{4}{3}(-1+\alpha)(1+\alpha)\left(1+\alpha^{2}\right)$ \\
& 3 & $\frac{1}{5}\left(1+10 \alpha^{2}+5 \alpha^{4}\right)$ & $\frac{1}{3}\left(3+\alpha^{2}\right)\left(1+3 \alpha^{2}\right)$ \\
\hline 4 & 1 & $-\frac{17}{315}(-1+\alpha)^{3}(1+\alpha)^{3}$ & $-\frac{62}{315}(-1+\alpha)^{3}(1+\alpha)^{3}$ \\
& 2 & $-\frac{11}{45}(-1+\alpha)^{2}(1+\alpha)^{2}\left(1+3 \alpha^{2}\right)$ & $-\frac{6}{5}(-1+\alpha)^{2}(1+\alpha)^{2}\left(1+\alpha^{2}\right)$ \\
& 3 & $-\frac{1}{3}(-1+\alpha)(1+\alpha)\left(1+10 \alpha^{2}+5 \alpha^{4}\right)$ & $-\frac{2}{3}(-1+\alpha)(1+\alpha)\left(3+\alpha^{2}\right)\left(1+3 \alpha^{2}\right)$ \\
& 4 & $-\frac{1}{7}\left(1+21 \alpha^{2}+35 \alpha^{4}+7 \alpha^{6}\right)$ & $-1\left(1+\alpha^{2}\right)\left(1+6 \alpha^{2}+\alpha^{4}\right)$ \\
\hline 5 & 1 & $\frac{62}{2835}(-1+\alpha)^{4}(1+\alpha)^{4}$ & $\frac{1382}{184175}(-1+\alpha)^{4}(1+\alpha)^{4}$ \\
& 2 & $\frac{88}{567}(-1+\alpha)^{3}(1+\alpha)^{3}\left(1+3 \alpha^{2}\right)$ & $\frac{848}{945}(-1+\alpha)^{3}(1+\alpha)^{3}\left(1+\alpha^{2}\right)$ \\
& 3 & $\frac{16}{45}(-1+\alpha)^{3}(1+\alpha)^{3}\left(1+10 \alpha^{2}+5 \alpha^{4}\right)$ & $\frac{37}{45}(-1+\alpha)^{2}(1+\alpha)^{2}\left(3+\alpha^{2}\right)\left(1+3 \alpha^{2}\right)$ \\
& 4 & $\frac{1}{3}(-1+\alpha)(1+\alpha)\left(1+21 \alpha^{2}+35 \alpha^{4}+7 \alpha^{6}\right)$ & $\frac{8}{3}(-1+\alpha)(1+\alpha)\left(1+\alpha^{2}\right)\left(1+6 \alpha^{2}+\alpha^{4}\right)$ \\
& 5 & $\frac{1}{9}\left(1+36 \alpha^{2}+126 \alpha^{4}+84 \alpha^{6}+9 \alpha^{8}\right)$ & $\frac{1}{5}\left(5+10 \alpha^{2}+\alpha^{4}\right)\left(1+10 \alpha^{2}+5 \alpha^{4}\right)$ \\
\hline
\end{tabular}

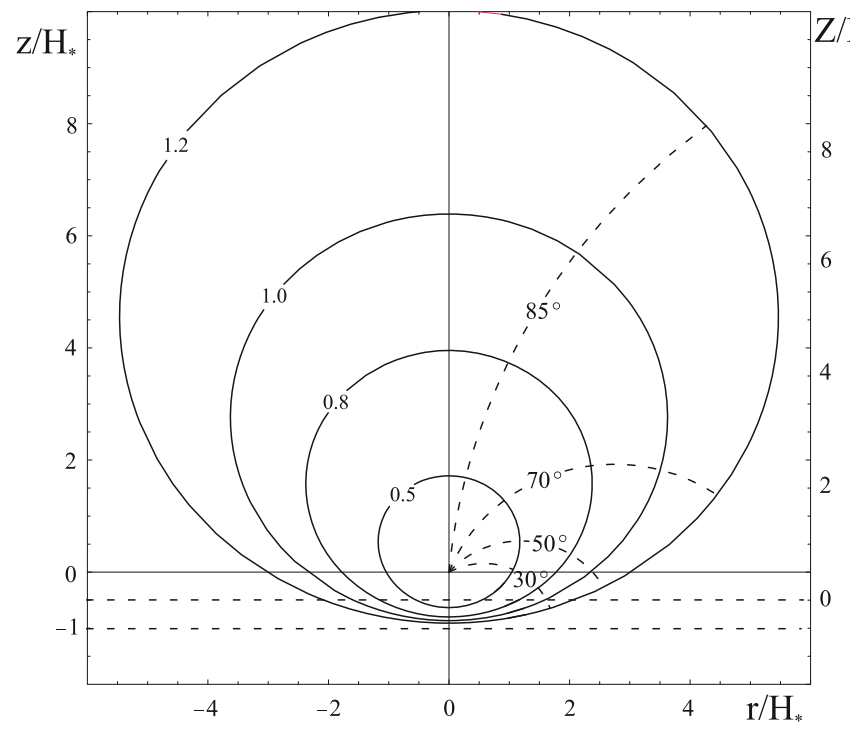

Fig. 4. Form of the shock wave (solid curves) at various evolutionary stages in a static gaseous medium with an initial density distribution given by the law: $\frac{1}{\left(1+\frac{Z}{Z}\right)^{2}}$. See caption of Fig. 1 for the meaning of the different symbols. The correspondence between $t_{\star}$, whose represented values are marked on the solid curves, and the elapsed time is given in Appendix B.

that the stars and gas in the Galactic disk are isothermal and self-gravitating, and solving the Poisson equation in hydrodynamical equilibrium, Spitzer (1942) obtained a quadratic hyperbolic-secant law for the dynamical $z$-distribution of stars and ISM (see also Rohlfs 1977). Hence, the gas density distribution of the Galactic disk can be represented by

$\rho=\rho_{\mathrm{c}} \operatorname{sech}^{2}(Z / H)$,

where $\rho_{\mathrm{c}}$ is the gas density in the Galactic plane, $Z=0$. Denoting by $Z_{0}$ the height of the explosion site and replacing $Z=Z_{0}+z$ in Eq. (34), taking into account that the density in the explosion site is therefore $\rho_{0}=\rho_{\mathrm{c}} \operatorname{sech}^{2}\left(Z_{0} / H\right)$, we obtain the density distribution with respect to $z$,
$F(z)=[\cosh (z / H)+\alpha \sinh (z / H)]^{-2}$, where $\alpha=\tanh \left(Z_{0} / H\right)$, see Eq. (20). We can express $F(z)^{-1}$ as the following power series: $F(z)^{-1}=1+\alpha \sum_{n=1}^{\infty} \frac{2^{4 n-2}}{\prod_{k=1}^{2 n-1} 2 k}(z / H)^{2 n-1}+\left(1+\alpha^{2}\right) \sum_{n=1}^{\infty} \frac{2^{2 n-1}}{(2 n) !}(z / H)^{2 n}$. By comparing this series expansion with Eq. (15), we determine the coefficients $f_{n}$, with which we obtain from Tables $1 \mathrm{a}$ and $1 \mathrm{~b}$ the expressions for $a_{r}(i, j), b_{r}(i, j), a_{z}(i, j)$ and $b_{z}(i, j)$. We see that $b_{z}(i, 1)=0, a_{r}(i, j)=a_{z}(i, j)$ and $b_{r}(i, j)=-b_{z}(i, j+1)$. Then we can conveniently rewrite Eqs. (18) and (19) as:

$$
\begin{aligned}
r= & 2 H \sum_{i=1}^{\infty}\left\{\left[\sum_{j=1}^{i} a_{r}^{\star}(i, j) \cos (2 j-1) \varphi\right] t_{\star}^{2 i-1}\right. \\
& \left.+\alpha\left[\sum_{j=1}^{i} b_{r}^{\star}(i, j) \sin 2 j \varphi\right] t_{\star}^{2 i}\right\}, \\
z= & 2 H \sum_{i=1}^{\infty}\left\{\left[\sum_{j=1}^{i} a_{z}^{\star}(i, j) \sin (2 j-1) \varphi\right] t_{\star}^{2 i-1}\right. \\
& \left.+\alpha\left[\sum_{j=1}^{i} b_{z}^{\star}(i, j) \cos 2 j \varphi\right] t_{\star}^{2 i}\right\},
\end{aligned}
$$

where $a_{r}^{\star}(i, j)=\frac{1}{(2 i-1) !} a_{r}(i, j)$ and $b_{r}^{\star}(i, j)=\frac{1}{\alpha(2 i) !} b_{r}(i, j+1)$. The expressions for $a_{r}^{\star}(i, j)$ and $b_{r}^{\star}(i, j)$ are given in Table 3 and, $a_{z}^{\star}(i, j)=a_{r}^{\star}(i, j)$ and $b_{z}^{\star}(i, j)=-b_{r}^{\star}(i, j)$.

Now we proceed to find the functions that reproduce the power series expansion of Eqs. (35) and (36). A guide is the trajectory of the upper point of the shock wave, which is linear $(\varphi=\pi / 2$ and always $r=0)$ and can be easily determined integrating Eq. (11) by the method of separation of variables. Assuming for simplicity that $\alpha=0$, i.e. an explosion in the Galactic plane, the result is: $z=2 H \operatorname{arctanh}\left(\tan t_{\star}\right)$. Using the identity $\operatorname{arctanh}\left(\tan t_{\star}\right)=\frac{1}{2} \ln \frac{1+\tan t_{\star}}{1-\tan t_{\star}}$, and since $\sin \varphi=1$ in this particular solution, we can guess that the solution for any $\varphi$ is

$z=2 H \frac{1}{2} \ln \frac{\sqrt{1+2 p \sin \varphi+p^{2}}}{\sqrt{1-2 p \sin \varphi+p^{2}}}$ 
where $p=\tan t_{\star}$. We arrive at the same conclusion analyzing the formula $\sum_{k=1}^{\infty}(-1)^{k-1} \frac{p^{2 k-1} \sin (2 k-1) x}{2 k-1}=\frac{1}{4} \ln \frac{1+2 p \sin x+p^{2}}{1-2 p \sin x+p^{2}}$ (Grandshteyn \& Ryzhik 2000) and comparing with Eq. (36) and the corresponding coefficients of Table 3. When $\alpha=0$, the second members of Eqs. (35) and (36), depending on $b_{r}^{\star}(i, j)$, are eliminated and the remaining members are odd functions of $t_{\star}$. On the other hand, the formula $\sum_{k=1}^{\infty}(-1)^{k-1} \frac{p^{2 k-1} \cos (2 k-1) x}{2 k-1}=$ $\frac{1}{2} \arctan \frac{2 p \cos x}{1-p^{2}}$ (Grandshteyn \& Ryzhik 2000) can be identified with Eq. (35), then the solution for $r$ when $\alpha=0$ is

$r=2 H \frac{1}{2} \arctan \frac{2 p \cos \varphi}{1-p^{2}}$,

where $p=\tan t_{\star}$. We need to find another exact particular solution for $z$ or $r$ to construct the general solution for $r$ and $z$. We start investigating a solution for $z$ and then proceeding by analogy we will find the solution for $r$. When $\alpha=1$, all terms of Table 3 become zero except those with indexes $j=i$. In this case, Eq. (36) gives $z=2 H\left\{\frac{1}{2} \sum_{i=1}^{\infty}(-1)^{i-1} \frac{\sin (2 i-1) \varphi}{2 i-1}\left(2 t_{\star}\right)^{2 i-1}+\right.$ $\left.\frac{1}{2} \sum_{i=1}^{\infty}(-1)^{i} \frac{\cos 2 i \varphi}{2 i}\left(2 t_{\star}\right)^{2 i}\right\}$, which corresponds to the solution

$$
\begin{aligned}
z= & 2 H\left\{\frac{1}{2}\left[\frac{1}{4} \ln \frac{1+2 p \sin \varphi+p^{2}}{1-2 p \sin \varphi+p^{2}}\right]\right. \\
& \left.+\frac{1}{2}\left[-\frac{1}{4} \ln \left(1-2 p \sin \varphi+p^{2}\right)-\frac{1}{4} \ln \left(1+2 p \sin \varphi+p^{2}\right)\right]\right\},
\end{aligned}
$$

where $p=2 t_{\star}$. Observe that the coefficients $a_{r}^{\star}(i, j)$ with $j=i$, determining this solution, can be reduced to the expression $\frac{2(-1)^{i+1}}{2 i-1}\left(\frac{1}{2}\left[(1+\alpha)^{i}+(1-\alpha)^{i}\right]\right.$ (see Table 3$)$. This indicates that $p \rightarrow(1+\alpha) t_{\star} \rightarrow 2 t_{\star}$ as $\alpha \rightarrow 1$. The sum of the term $(1-\alpha)^{i}$ in this expression also indicates that some terms of the general solution depend on a function $q \rightarrow(1-\alpha) t_{\star}$ as $\alpha \rightarrow 1$. Therefore, based on Eqs. (37) and (39), we make the following ansatz for the general solution for $z$ :

$$
\begin{aligned}
z= & 2 H\left[\frac{1}{2}\left\{\frac{1}{4} \ln \frac{1+2 p \sin \varphi+p^{2}}{1-2 p \sin \varphi+p^{2}}\right\}+\frac{1}{2}\left\{\frac{1}{4} \ln \frac{1+2 q \sin \varphi+q^{2}}{1-2 q \sin \varphi+q^{2}}\right\}\right. \\
& +\frac{1}{2}\left\{-\frac{1}{4} \ln \left(1-2 p \sin \varphi+p^{2}\right)-\frac{1}{4} \ln \left(1+2 p \sin \varphi+p^{2}\right)\right\} \\
& \left.-\frac{1}{2}\left\{-\frac{1}{4} \ln \left(1-2 q \sin \varphi+q^{2}\right)-\frac{1}{4} \ln \left(1+2 q \sin \varphi+q^{2}\right)\right\}\right] .
\end{aligned}
$$

If $p=q=\tan t_{\star}$, we obtain the particular solution of Eq. (37); and if $p=2 t_{\star}$ and $q=0$, we obtain the particular solution of Eq. (39). Similarly, we propose the following general solution for $r$ :

$$
\begin{aligned}
r= & 2 H\left\{\frac{1}{2}\left(\frac{1}{2} \arctan \frac{2 p \cos \varphi}{1-p^{2}}+\frac{1}{2} \arctan \frac{2 q \cos \varphi}{1-q^{2}}\right)\right. \\
& +\frac{1}{2}\left(\arctan \frac{p \cos \varphi}{1-p \sin \varphi}-\frac{1}{2} \arctan \frac{2 p \cos \varphi}{1-p^{2}}\right) \\
& \left.-\frac{1}{2}\left(\arctan \frac{q \cos \varphi}{1-q \sin \varphi}-\frac{1}{2} \arctan \frac{2 q \cos \varphi}{1-q^{2}}\right)\right\} .
\end{aligned}
$$

We assume that $p$ and $q$ have the form: $p=(1+\alpha) t_{\star}+p_{3} t_{\star}^{3}+$ $p_{5} t_{\star}^{5}+\ldots$ and $q=(1-\alpha) t_{\star}+q_{3} t_{\star}^{3}+q_{5} t_{\star}^{5}+\ldots$ We insert these expressions for $p$ and $q$ in Eq. (40) or Eq. (41), which is then expanded in power series of $t_{\star}$. Comparing the first terms that depend on $p_{3}$ and $q_{3}$ with the corresponding ones of Eq. (36) or Eq. (35), i.e. terms of the same power of $t_{\star}$, we obtain the values of $p_{3}$ and $q_{3}$. We substitute the values found for $p_{3}$ and $q_{3}$, and repeat the procedure to find $p_{5}$ and $q_{5}$, and so on. The result is:

$$
\begin{aligned}
p= & (1+\alpha) t_{\star} \\
& -\frac{1}{3}(-1+\alpha)(1+\alpha)^{2} t_{\star}^{3}+\frac{2}{15}(-1+\alpha)^{2}(1+\alpha)^{3} t_{\star}^{5}-\ldots \\
= & (1+\alpha) t_{\star}+\sum_{n=2}^{\infty}(-1+\alpha)^{n-1}(1+\alpha)^{n} B_{2 n} \frac{\left(-4^{n}\right)\left(1-4^{n}\right)}{(2 n) !} t_{\star}^{2 n-1} \\
q= & -(-1+\alpha) t_{\star}+\frac{1}{3}(-1+\alpha)^{2}(1+\alpha) t_{\star}^{3} \\
& -\frac{2}{15}(-1+\alpha)^{3}(1+\alpha)^{2} t_{\star}^{5}+\ldots \\
= & -(-1+\alpha) t_{\star}-\sum_{n=2}^{\infty}(-1+\alpha)^{n}(1+\alpha)^{n-1} \\
& \times B_{2 n} \frac{\left(-4^{n}\right)\left(1-4^{n}\right)}{(2 n) !} t_{\star}^{2 n-1}
\end{aligned}
$$

where $B_{n}$ are the Bernoulli numbers. Equations (40) and (41), arrayed to show the symmetry of the solutions, can be written in simplified form as:

$$
\begin{aligned}
& r=2 H\left\{\frac{1}{2}\left[\arctan \frac{(p+q) \cos \varphi}{1-p q-(p-q) \sin \varphi}\right]\right\}, \\
& z=2 H\left(\frac{1}{4} \ln \frac{1+2 q \sin \varphi+q^{2}}{1-2 p \sin \varphi+p^{2}}\right) .
\end{aligned}
$$

Equation (44) was obtained simplifying Eq. (41) by means of the identity $\arctan x \pm \arctan y=\arctan \frac{x \pm y}{1 \mp x y}$. Summarizing, Eqs. (44) and (45) with $p$ and $q$ given by Eqs. (42) and (43) provide an analytical solution to the problem of the propagation of a strong shock in a gaseous disk whose density distribution in $Z$ follows the $\operatorname{sech}^{2} Z$ law. These equations can be used for any position of the explosion point within the disk, and describe the evolution of each point of the shock wave, wherever in the disk the wave reaches. In Fig. 5, we apply these equations to the case of an explosion outside the Galactic plane or, speaking in general terms, outside the middle plane of a disk, whose plane is defined by the maximum of density $\rho_{\mathrm{c}}$ (Eq. (34)). Since Eq. (34) is an even function, this represents the density distribution at both sides of the middle plane, that is to say for $Z>0$ as well as for $Z<0$, and we do not need to distinguish between these subspaces as in the exponential model (Sect. 4.1). Hence, in the framework of the Kompaneets approximation, Eqs. (44) and (45) give us a complete description of the propagation of the wave in the disk.

The time $t_{\star}$ at which the bubble created by the shock wave blows out of the disk is here denoted by $t_{b}$. In Fig. 6, we plot $t_{b}$ as a function of $\alpha$ or $Z_{0} / H$. Equation (45) shows that, for $\alpha>0, z$ of the top of the wave $(\varphi=\pi / 2)$ tends to infinity when $p=1$ and, for $\alpha<0$ and $\varphi=-\pi / 2$, when $q=1$. Then equating Eq. (42) or Eq. (43) to 1 and solving for $t_{\star}$, we obtain $t_{b}$. If $\alpha=0$, an explosion in the midplane, then $p=q=\tan t_{\star}=1$ and hence $t_{b}=0.78$.

We can eliminate from Eqs. (44) and (45) the $\sin \varphi$ and $\cos \varphi$ functions and obtain the canonical equation of the surface of the wave as a function of time. Because this general equation is rather complicated and has little practical value, we do not 


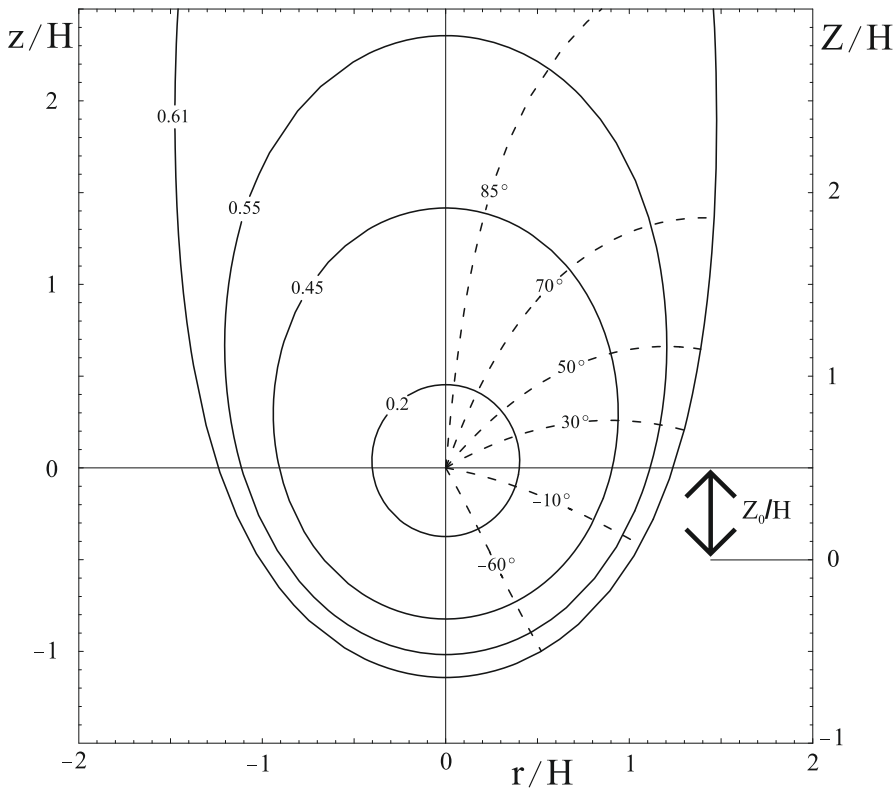

Fig. 5. Shock wave propagating in a static gaseous medium with $\operatorname{sech}^{2}(Z / H)$ density distribution, referred to the explosion point (left vertical axis), whose off-position with respect to the Galactic plane (or central plane of symmetry) is $Z_{0} / H=0.5$, i.e. $\alpha=0.462$. Its form (solid curves) is shown at different $t_{\star}:\left\{t_{i}\right\}=\{0.2,0.45,0.55,0.61\}$. We show the stream lines (dashes lines) corresponding to the set of angles $\left\{\varphi_{i}\right\}=\left\{-60^{\circ},-10^{\circ}, 30^{\circ}, 50^{\circ}, 70^{\circ}, 85^{\circ}\right\}$. The right hand vertical axis shows the coordinate $Z$ referred to the Galactic plane. The correspondence between $t_{\star}$ and the elapsed time $t$ is given in Appendix B (see also Figs. B.3 and B.4).

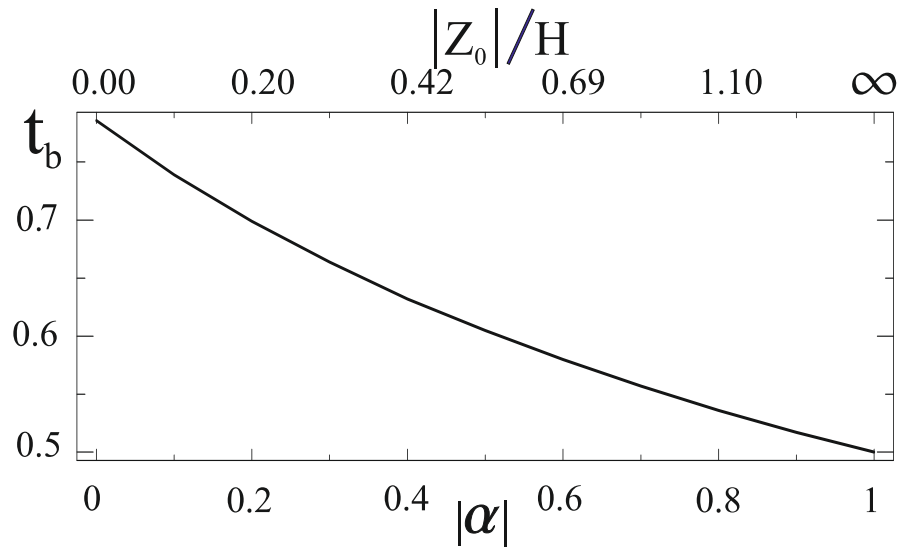

Fig. 6. Dependence of the blow out time $t_{b}$ with $\alpha$ (lower horizontal scale) or $Z_{0}$ (upper horizontal scale) for the wave in a medium of $\operatorname{sech}^{2}(Z / H)$ density distribution. $t_{b}$ is given by the particular value of the parameter $t_{\star}$ at which the top of the wave front formally reaches infinity. $Z_{0}$ is the height of the blast point and $\alpha=\tanh \frac{Z_{0}}{H}$.

give it here. However, it is instructive to obtain this equation for a particular case, viz., when the explosion occurs in the symmetry plane, i.e. $\alpha=0$. Here $p=q=\tan t_{\star}$ and Eqs. (44) and (45) are simplified. Solving Eq. (45) for $\sin \varphi$ and Eq. (44) for $\cos \varphi$, and using the identity $\sin ^{2} \varphi+\cos ^{2} \varphi=1$, we get

$r=H \arctan \sqrt{\tan ^{2} 2 t_{\star}-\sec ^{2} 2 t_{\star} \tanh ^{2} \frac{z}{H}}$.

It is easy to verify that Eq. (46) satisfies the Kompaneets equation (Eq. (9)). Eliminating $t_{\star}$ from Eqs. (44) and (45), we obtain

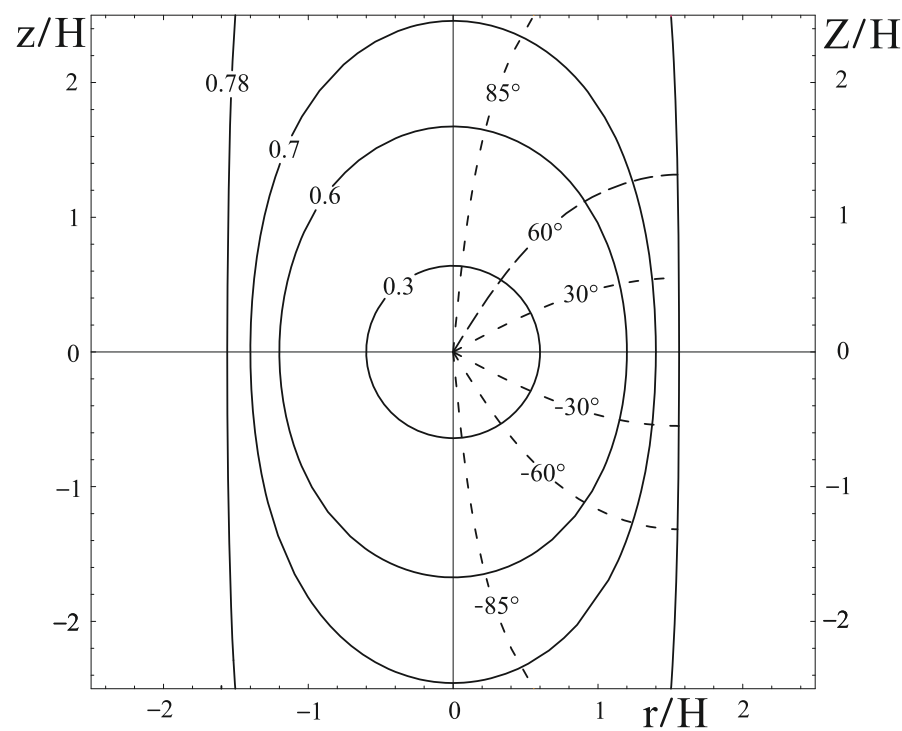

Fig. 7. Evolution of a strong shock wave originated by a point explosion in the midplane of a disk with a $\operatorname{sech}^{2}(Z / H)$ law of density distribution. Setting $\alpha=0$ and the time $t_{\star}$ to the desired value, we obtain from Eqs. (44) and (45) the set of points $\{r(\varphi), z(\varphi)\}$, with $\varphi$ as the independent variable ranging from 0 to $2 \pi$, representing the surface of the wave at the chosen time (full curves with times marked). Similarly but fixing $\varphi$ to the desired value, we obtain the set of points $\left\{r\left(t_{\star}\right), z\left(t_{\star}\right)\right\}$, representing the orbit of the point identified with the chosen $\varphi$ (dashed lines with angles marked on). The correspondence between $t_{\star}$ and the elapsed time $t$ is given in Appendix B (see also Figs. B.3 and B.4).

the equation for the orbits of the individual points (streamlines), but again it is easier to compute them by using the equations in parametric form. The case we have been treating in this paragraph, the evolution of a strong shock wave originating in the midplane of a disk (i.e. $\alpha=0$ ), is illustrated in Fig. 7.

\section{Summary and conclusions}

In the light of the Kompaneets approximation, we were able to find analytical solutions for the propagation of a shock wave in different stratified media. We considered four plane-parallel stratified media, including the barometric atmosphere adopted in the Kompaneets model, whose density-distribution laws are summarized in Table 4. We adapted the Kompaneets equations that govern the propagation of the wave in order to obtain solutions in parametric form (Col. 3 of Table 4). The adapted Kompaneets equations were solved via an analytical approach based on a power-series method, and the exact functions that reproduce the power-series solutions were identified.

Though the problem was initially posed for the case of a point explosion in which all of the kinetic energy is released to the environment almost instantaneously, our solutions can be also apply to the case of continuous injection of energy (Schiano 1985; Basu et al. 1999). Note that Eq. (8) admits a function of time for the energy that the source releases. Thus, in an astrophysical context, our solutions permit us to study the evolution of a bubble created by a supernova explosion, as well as that of a bubble insufflated by stellar winds and/or multiple explosions.

Out of the four considered media, the medium of the $\operatorname{sech}^{2} Z$ density distribution is the most appropriate one to represent the overall vertical distribution of the ISM in the Galactic disk. There are dynamical reasons (Spitzer 1942; Rohlfs 1977) and observational ones (Dickey \& Lockman 1990; 
Table 4. Synopsis of the basic equations that characterize the studied gas media and the propagation of a shock wave within them ${ }^{a}$.

\begin{tabular}{|c|c|c|c|}
\hline$\rho(Z)=$ & $\frac{\rho(z)}{\rho_{0}}=F(z)=$ & Solutions for the shock-wave motion & Blow-out time $t_{b}$ \\
\hline$\rho_{\mathrm{c}} \exp (-Z / H)$ & $\exp (-z / H)$ & $\begin{array}{l}r=2 H \arctan \left(\frac{t_{\star} \cos \varphi}{1-t_{\star} \sin \varphi}\right) \\
z=-2 H \ln \left(\sqrt{1-2 t_{\star} \sin \varphi+t_{\star}^{2}}\right)\end{array}$ & 1.0 \\
\hline for $0 \leq Z \leq \infty$ & for $-Z_{0} \leq z \leq \infty$ & for $-Z_{0} \leq z \leq \infty$ & \\
\hline$\frac{\rho_{\mathrm{c}}}{\left(1+\frac{Z}{H}\right)}$ & $\begin{array}{l}\frac{1}{\left(1+\frac{z}{H_{\star}}\right)} \\
\text { where } H_{\star}=H+Z_{0}\end{array}$ & $\begin{array}{l}r=2 H_{\star}\left\{t_{\star} \cos \varphi+\frac{\cos 2 \varphi}{(2 \cos \varphi)^{2}}\left[\sin \left(2 t_{\star} \cos \varphi\right)-2 t_{\star} \cos \varphi\right)\right]+ \\
\left.\frac{\sin \varphi}{2 \cos \varphi}\left[1-\cos \left(2 t_{\star} \cos \varphi\right)\right]\right\} \\
z=2 H_{\star}\left\{\frac{\sin 2 \varphi}{2 \cos \varphi} \sin \left(2 t_{\star} \cos \varphi\right)+\frac{\cos 2 \varphi}{(2 \cos \varphi)^{2}}\left[\cos \left(2 t_{\star} \cos \varphi\right)-1\right]\right\}\end{array}$ & $\infty$ \\
\hline for $0 \leq Z \leq \infty$ & for $-Z_{0} \leq z \leq \infty$ & for $-Z_{0} \leq z \leq \infty$ & \\
\hline $\begin{array}{l}\frac{\rho_{\mathrm{C}}}{\left(1+\frac{Z}{H}\right)^{2}} \\
\text { for } 0 \leq Z \leq \infty\end{array}$ & $\begin{array}{l}\frac{1}{\left(1+\frac{z}{H_{\star}}\right)^{2}} \\
\text { where } H_{\star}=H+Z_{0} \\
\text { for }-Z_{0} \leq z \leq \infty\end{array}$ & $\begin{array}{l}r=H_{\star} \frac{\cos \varphi}{\operatorname{coth} 2 t_{\star}-\sin \varphi} \\
z=H_{\star} \frac{2 \sin \varphi-\cos ^{2} \varphi \sinh 2 t_{\star}}{\operatorname{coth} t_{\star}-\sin \varphi+\cos ^{2} \varphi \sinh 2 t_{\star}} \\
\text { for }-Z_{0} \leq z \leq \infty\end{array}$ & $\infty$ \\
\hline for $-\infty \leq Z \leq \infty$ & $\begin{array}{l}{[\cosh (z / H)+\alpha \sinh (z / H)]^{-2}} \\
\text { where } \alpha=\tanh \left(Z_{0} / H\right)\end{array}$ & $\begin{array}{l}r=H \arctan \frac{(p+q) \cos \varphi}{1-p q-(p-q) \sin \varphi} \\
z=H \ln \sqrt{\frac{1+2 q \sin \varphi+q^{2}}{1-2 p \sin \varphi+p^{2}}} . \\
\text { where } p=(1+\alpha) t_{\star}+\sum_{n=2}^{\infty}(-1+\alpha)^{n-1}(1+\alpha)^{n} B_{2 n} \frac{\left(-4^{n}\right)\left(1-4^{n}\right)}{(2 n) !} t_{\star}^{2 n-1} \\
\text { and, } q=-(-1+\alpha) t_{\star}-\sum_{n=2}^{\infty}(-1+\alpha)^{n}(1+\alpha)^{n-1} B_{2 n} \frac{\left(-4^{n}\right)\left(1-4^{n}\right)}{(2 n) !} t_{\star}^{2 n-1} . \\
\text { for }-\infty \leq z \leq \infty\end{array}$ & $0.5-0.78$ \\
\hline
\end{tabular}

${ }^{a}$ Density distribution of the plane-parallel stratified medium, with respect to a $Z$ axis (Col. 1) and to a $z$ axis (Col. 2). The $z$ and $Z$ axes are along the line that passes through the explosion point and is perpendicular to the stratification plane. The origin of $Z$-axis lies on the plane of maximum gas density $\rho_{\mathrm{c}}$ or symmetry plane, e.g. the Galactic plane. Both positive axes point in the same sense, toward decreasing densities. We denote the $Z$-position (or altitude) of the explosion point by $Z_{0}$, origin of the $z$ axis. The positions $(r, z)$ of the wave, given by the parametric equations $(C o l .3$ ), are referred to the cylindrical coordinate system $(r, z)$ with origin in the explosion point and symmetry around the $z$-axis. The valid range for each function is given.

Marshall et al. 2006) that favor the election of the quadratic hyperbolic-secant distribution for galactic studies. The $\operatorname{sech}^{2} Z$ goes over into the Gaussian for small $Z$, and for large $Z$, declines slower that the Gaussian. On the other hand, the use of the $\operatorname{sech}^{2} Z$ distribution permitted us to find, in the framework of the Kompaneets approximation, the most general solution for the propagation of a shock wave in a medium such as the Galactic disk, without having to numerically solve the complete system of hydrodynamic equations. Therefore the solution derived from the Kompaneets approximation (the bottom row of Table 4) is an important tool for studying Galactic shells and supershells (Heiles 1979), originating in stellar energy sources as well as impacts of high-velocity clouds on the Galactic disk (TenorioTagle 1980, 1981; Olano 2004, 2008).

If a shell reaches radii larger than the scale height of the Galactic gaseous disk (see Figs. 5 and 7), we can call it a supershell or shell of a superbubble. By the time of the blow out, the internal pressure of a superbubble drops to a very low value and other forces begin to dominate the motion of the associated supershell. The gravity restoring force in the Z-direction, the differential rotation of the Galactic disk and the resistive forces due to the interaction of the expanding supershell with the surrounding ISM govern the later evolutionary stages of the supershell (e.g. Olano 1982). The solutions obtained from the Kompaneets approximation can give not only the shape of the supershell at a certain time, but also the velocity and column density for each piece of the supershell's surface (see Appendix B). Then these calculations can provide the initial conditions to model the later evolutionary stages of these superstructures. The application of other approximate analytical methods (e.g. Hnatyk \& Petruk 1999), of the thin-shell approximation (e.g. Mac Low \& McCray 1988; Silich 1992) and of the full numerical calculations (e.g. Mac Low et al. 1989) to the shock wave propagation in each of the stratified media we studied here by means of the Kompaneets approximation, would permit us to make an interesting comparative study.

Acknowledgements. I dedicate this work to the memory of Dr. F. Raúl Colomb (1939-2008), one of the pioneers of the radioastronomy and space activities in Argentina, with who I shared an interest in astronomical theories of terrestrial catastrophism. My thanks to the anonymous referee for helpful comments which helped me to improve the manuscript. Part of this work was supported by the Consejo Nacional de Investigaciones Científicas y Técnicas (CONICET) project number PIP112-200801-0248.

\section{Appendix A: calculation of the coefficients of the power series}

The conditions that $c_{n}=0$ and $d_{n}=0$ allow us to obtain the solutions for $r_{n}(\varphi)$ and $z_{n}(\varphi)$ (see Sect. 2). The explicit expressions of the coefficients $c_{0}$ and $d_{1}$ are: $c_{0}=r_{1}^{2}+z_{1}^{2}-4 H^{2}=0$ and $d_{1}=r_{1} \dot{r}_{1}+z_{1} \dot{z}_{1}=0$, which are satisfied by the following solution,
$r_{1}=2 H \cos \varphi$
$z_{1}=2 H \sin \varphi$.

The following pair of coefficients $c_{1}$ and $d_{2}$ are given by $c_{1}=$ $r_{1} r_{2}+z_{1}\left(-f_{1} H+z_{2}\right)=0$ and $d_{2}=2 r_{2} \dot{r}_{1}+r_{1} \dot{r}_{2}+2 z_{2} \dot{z}_{1}+z_{1} \dot{z}_{2}=0$. Solving $c_{1}$ for $r_{2}$, after substituting Eqs. (A.1), we find

$r_{2}=\left(f_{1} H-z_{2}\right) \tan \varphi$ 
and hence,

$\dot{r}_{2}=\left(f_{1} H-z_{2}\right) \sec ^{2} \varphi-z_{2} \tan \varphi$.

Replacing Eqs. (A.2), (A.3) and (A.1) in $d_{2}$, then we have $d_{2}=$ $2 H\left(z_{2}+f_{1} H \cos 2 \varphi\right) \sec \varphi=0$. Note that the terms depending on $\dot{z}_{2}$ are cancelled. Solving $d_{2}$ for $z_{2}$ and substituting this result in Eq. (A.2) we obtain

$r_{2}=f_{1} H \sin 2 \varphi$

$z_{2}=-f_{1} H \cos 2 \varphi$.

Repeating the procedure for $c_{2}=4 r_{2}^{2}+6 r_{1} r_{3}-4 f_{2} z_{1}^{2}-4 f_{1} H z_{2}+$ $4 z_{2}^{2}+6 z_{1} z_{3}=0$ and $d_{3}=3 r_{3} \dot{r}_{1}+2 r_{2} \dot{r}_{2}+r_{1} \dot{r}_{3}+3 z_{3} \dot{z}_{1}+2 z_{2} \dot{z}_{2}+z_{1} \dot{z}_{3}=$ 0 , we obtain $r_{3}$ and $z_{3}$. Proceeding in a similar form, we find the solutions for the rest of the coefficients.

\section{Appendix B: Applications of the solutions}

As a consequence of the propagation of a shock front, a thin shell of material swept from the surrounding gaseous medium is formed just behind the shock front. Hence, given an observed structure that can be interpreted as a shell enclosing a cavity we can apply our solutions to it. Having specified the gaseous medium in which the shell is immersed, the shape of the shell is completely described in terms of the dimensionless parameter $t_{\star}$. Fitting the theoretical shape of the shell to the observed shape, one obtains $t_{\star}$ and even the age $t$ of the shell (if one has estimates for the energy $E$ of the explosion, the density near the explosion site $\rho_{0}$ and the scale height $H$ of the medium ). Inverting the transformation of Eq. (8) and recalling that we defined $y=2 H t_{\star}$, we can obtain the elapsed time $t$ as a function of $t_{\star}$. The total volume included inside the shell can be written $V\left(t_{\star}\right)=\pi \int_{z 1}^{z 2} r^{2} \mathrm{~d} z=\pi H^{3} \Omega\left(t_{\star}\right)$, where

$\Omega\left(t_{\star}\right)=\int_{-\pi / 2}^{\pi / 2}(r / H)^{2} \frac{\partial(z / H)}{\partial \varphi} \mathrm{d} \varphi$.

Hence Eq. (8) implies that

$\mathrm{d} t=\tau \sqrt{\Omega\left(t_{\star}\right)} \mathrm{d} t_{\star}$

and

$t=\tau \int_{0}^{t_{\star}} \sqrt{\Omega\left(t_{\star}\right)} \mathrm{d} t_{\star}$,

where $\tau=\sqrt{\frac{8 \pi H^{5} \rho_{0}}{\lambda E\left(\gamma^{2}-1\right)}}$ (cf. Kompaneets 1960).

The velocity of a point of the shock front $\boldsymbol{v}_{\star}=\left(\frac{\partial r}{\partial t_{\star}}, \frac{\partial z}{\partial t_{\star}}\right)$, as obtained from the formulae of the chosen atmosphere (Col. 3 of Table 4), is expressed in units of length/time by means of $\boldsymbol{v}=\boldsymbol{v}_{\star} \frac{\mathrm{d} t_{\star}}{\mathrm{d} t}=\left(\frac{\partial r}{\partial t_{\star}} \frac{\mathrm{d} t_{\star}}{\mathrm{d} t}, \frac{\partial z}{\partial t_{\star}} \frac{\mathrm{d} t_{\star}}{\mathrm{d} t}\right)$, where $\frac{\mathrm{d} t_{\star}}{\mathrm{d} t}=\frac{1}{\tau \sqrt{\Omega\left(t_{\star}\right)}}$, in accordance with Eq. (B.1). The relation between the velocity of a surface element of the shock front and the mean velocity of the disturbed gas behind this part of the shock front is not considered in the Kompaneets model. However, we can say from general considerations that in the adiabatic phase and even more in the radiative phase, when the associated shell of shocked gas cools radiatively and becomes thinner, the mean gas velocity of a part of the shell tends to be equal to the velocity of the associated part of the shock front (Bisnovatyi-Kogan \& Silich 1995).

Our formulae permit us also to calculate the column density $\eta\left(t_{\star}, \varphi\right)$ for each point and evolutionary stage of the shell. The

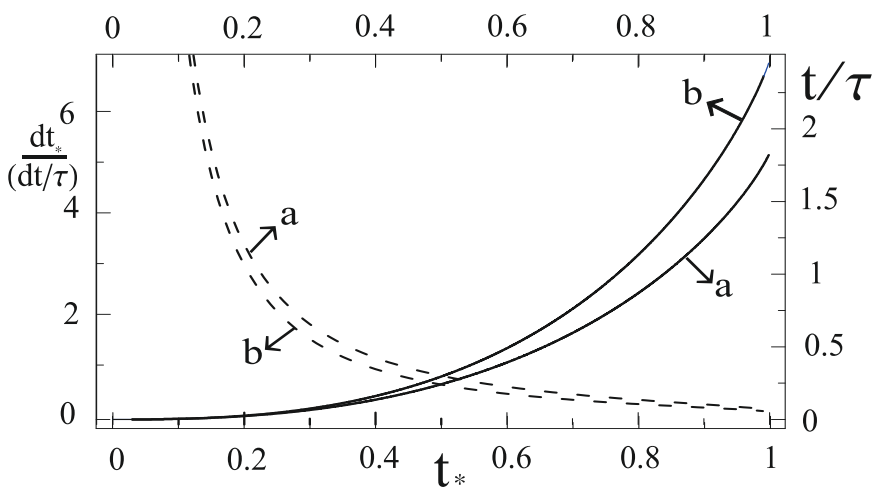

Fig. B.1. Relationship between $t_{\star}$ and $t / \tau$ (full curves and right vertical scale), and relationship between $t_{\star}$ and $\frac{\mathrm{d} t_{\star}}{\mathrm{d} t / \tau}$ (dashed curves and left vertical scale), for shells in exponential atmospheres. The symbol "a" labels the curves corresponding to an infinite medium with a single exponential density distribution (see Fig. 1), and "b" to a disk with a double exponential density distribution in whose symmetry plane is located the explosion point (see Fig. 2).

increment of the mass accumulated by an element of surface $\mathrm{d} A$ of the front moving along a differential of path, $\mathrm{d} s=\left|\boldsymbol{v}_{\star}\right| \mathrm{d} t_{\star}$, along the orbit characterized by $\varphi$ is $\rho_{0} F(z)\left|v_{\star}\right| \mathrm{d} t_{\star} \mathrm{d} A$. Although $\mathrm{d} A$ has always the same number of streamlines, the area of $\mathrm{d} A$ increases with time because of the divergence of the streamlines. The vector $\left(\frac{\partial r}{\partial \varphi}, \frac{\partial z}{\partial \varphi}\right)$ is perpendicular to the velocity vector $\boldsymbol{v}$ (see Sect. 2). Hence, the length of the sides of $\mathrm{d} A$ lying between the streamlines $\varphi$ and $\varphi+\mathrm{d} \varphi$ is $\sqrt{\left(\frac{\partial z}{\partial \varphi}\right)^{2}+\left(\frac{\partial r}{\partial \varphi}\right)^{2}} \mathrm{~d} \varphi$. The length of the other two sides of $\mathrm{d} A$, lying between the azimuthal angles $\Phi$ and $\Phi+\mathrm{d} \Phi$, is $r \mathrm{~d} \Phi$. Therefore the columnar density on each element of surface of the shell, defined by $\eta\left(t_{\star}, \varphi\right)=\frac{\mathrm{d} m\left(t_{\star}, \varphi\right)}{\mathrm{d} A\left(t_{\star}, \varphi\right)}$, is given by

$\eta=\rho_{0} H \frac{\int_{0}^{t_{\star}} F(z) r \sqrt{\left(\frac{\partial(z / H)}{\partial t_{\star}}\right)^{2}+\left(\frac{\partial(r / H)}{\partial t_{\star}}\right)^{2}} \sqrt{\left(\frac{\partial z}{\partial \varphi}\right)^{2}+\left(\frac{\partial r}{\partial \varphi}\right)^{2}} \mathrm{~d} t_{\star}}{r \sqrt{\left(\frac{\partial z}{\partial \varphi}\right)^{2}+\left(\frac{\partial r}{\partial \varphi}\right)^{2}}}$.

The integral of Eq. (B.3) can be solved analytically with the solutions for a shell formed in an atmosphere of a powerlaw density distribution (Sects. 4.2 and 4.3). Using the limaçon of Pascal as an approximation to the equation of the surface of the shell (see Sect. 4.2), $\Omega\left(t_{\star}\right)=\pi\left(4 t_{\star}^{3}+\frac{3}{4} t_{\star}^{5}\right)$ and $t=\frac{4}{5} \sqrt{\pi} t_{\star}^{5 / 2}{ }_{2} F_{1}\left(-\frac{1}{2}, \frac{5}{4}, \frac{9}{4},-\frac{3}{16} t_{\star}^{2}\right)$, where ${ }_{2} F_{1}$ refers to the corresponding hypergeometric function. Thus the set $t_{\star}=$ $\{0.5,1.0,1.5,2.0\}$ of the configurations represented in Fig. 3 is converted to the set of ages $t=\{0.25 \tau, 1.49 \tau, 4.34 \tau, 9.52 \tau\}$. In the case of the second atmosphere in which the shell's configurations are spherical with radii $\rho=H_{\star} \sinh 2 t_{\star}$ (see Sect. 4.3), $\Omega\left(t_{\star}\right)=\frac{4}{3}\left(\frac{\rho}{H_{\star}}\right)^{3}$ and the integral of Eq. (B.3) can be expressed in terms of a hypergeometric function or naturally solved numerically. Thus the conversion of the set $t_{\star}=\{0.5,0.8,1.0,1.2\}$ of Fig. 4 to ages is $t=$ $\{0.20 \tau, 1.06 \tau, 2.24 \tau, 4.45 \tau\}$, respectively. Applied to this last case, Eq. (B.4) yields a simple analytic result, namely: $\eta\left(t_{\star}, \varphi\right)=$ $\mathrm{d} m / \mathrm{d} A$, where $\mathrm{d} m=\rho_{0} H_{\star}\left[-\left(\sec ^{2} \varphi\right) \arctan \left(\sec \varphi \sinh 2 t_{\star}-\right.\right.$ $\left.\left.\cosh 2 t_{\star} \tan \varphi\right)+\sec \varphi \sinh 2 t_{\star}-\varphi \sec ^{2} \varphi+2 \sinh ^{2} t_{\star} \tan \varphi\right]$ and $\mathrm{d} A=\cos \varphi /\left(\cosh 2 t_{\star}-\sin \varphi\right)^{2}$. To refer this column density to the elevation angle $\phi$ measured relative to the $r$-axis, which agrees with $\varphi$ at $t_{\star}=0$, we can use the correspondence between both angles given in this case by $\tan \phi=\frac{z}{r}=\tan \varphi-\sec \varphi \tanh t_{\star}$. 


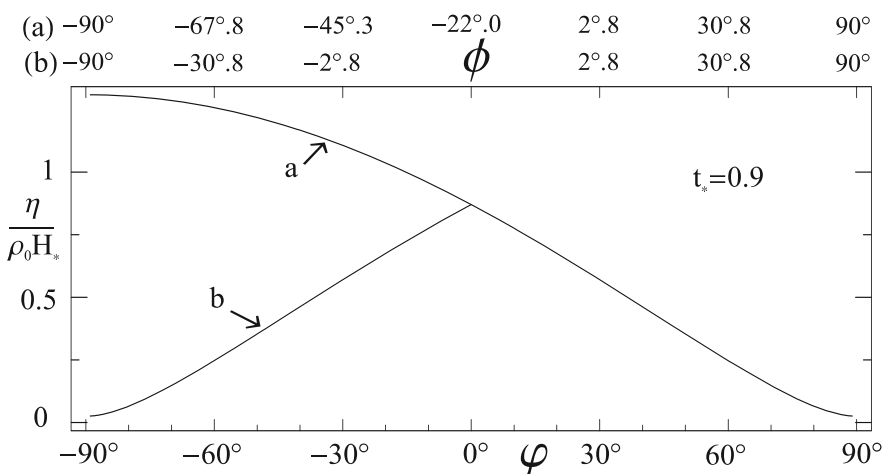

Fig. B.2. Relationship between $\varphi$ and $\eta / \rho_{0} H$ for shells in exponential atmospheres and $t_{\star}=0.9$. The upper horizontal scales give the corresponding elevation angles. The symbols "a" and "b" mean the same as in Fig. B.1.

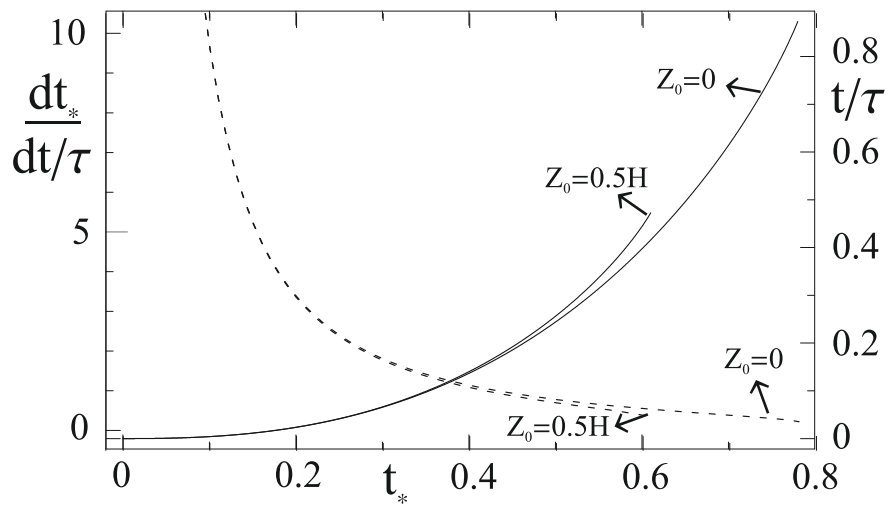

Fig. B.3. Relationship between $t_{\star}$ and $t / \tau$ (full curves and right vertical scale), and relationship between $t_{\star}$ and $\frac{\mathrm{d} t_{\star}}{\mathrm{d} t / \tau}$ for shells in media with $\operatorname{sech}^{2}(Z / H)$ density distribution. The curves for two positions of the explosion point, $Z_{0}=0$ (i.e. $\alpha=0$, see Fig. 7) and $Z_{0} / H=0.5$ (i.e. $\alpha=0.462$, see Fig. 5), are represented.

We will now proceed to evaluate numerically the integrals of Eqs. (B.1) and (B.3) with the solutions for the evolution of shells formed within atmospheres with exponential and $\mathrm{sech}^{2}$ density distributions (see Sects. 4.1 and 5). Thus we derive the curves of Figs. B.1 and B.3, relating $t_{\star}$ to $t / \tau$ and to $\frac{\mathrm{d} t_{\star}}{\mathrm{d} t / \tau}$. For the case "b" of Fig. B.1, the lower limit of integration is zero and the result of this integration is multiplied by 2 . Transforming $t_{\star}$ into $t$ by means of the curves of Fig. B.1, we find that the sequence of configurations represented in Figs. 1 and 2 , corresponding to the set $t_{\star}=\{0.3,0.6,0.8,0.9,1.0\}$, have the ages $t=\{0.07 \tau, 0.39 \tau, 0.87 \tau, 1.25 \tau, 1.85 \tau\}$ and $t=$ $\{0.07 \tau, 0.49 \tau, 1.14 \tau, 1.65 \tau, 2.45 \tau\}$, respectively. From the curves of Fig. B.3, we get that the stages of evolution represented by $t_{\star}=\{0.2,0.45,0.55,0.61\}$ (Fig. 5) and $t_{\star}=\{0.3,0.6,0.7,0.78\}$ (Fig. 7) have the ages $t=\{0.02 \tau, 0.19 \tau, 0.34 \tau, 0.47 \tau\}$ and $t=\{0.06 \tau, 0.40 \tau, 0.61 \tau, 0.87 \tau\}$, respectively. Solving numerically the integral in Eq. (B.4), we can estimate in units of $\rho_{0} H$ the column densities on the surface of a swept-up shell that evolved in a medium with exponential (Fig. B.2) and with $\operatorname{sech}^{2}$ density distribution (Fig. B.4) to a certain stage $t_{\star}$.

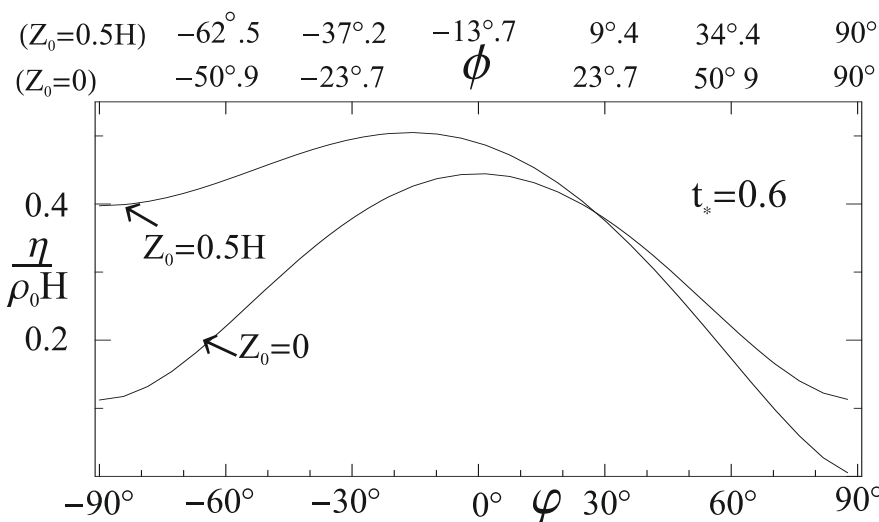

Fig. B.4. Column density (in units of $\rho_{0} H$ ) vs. angle $\varphi$ (or elevation angle $\phi$, using the upper horizontal scale) for the shell represented in Fig. $5\left(Z_{0}=0.5 H\right)$ and for the shell represented in Fig. $7\left(Z_{0}=0\right)$, when $t_{\star}=0.6$.

\section{References}

Basu, S., Johnstone, D., \& Martin, P. G. 1999, ApJ, 516, 843

Bisnovatyi-Kogan, G., \& Silich, S. 1995, Rev. Mod. Phys., 67, 611

Dickey, J. M.. \& Lockman, F. J. 1999, ARA\&A, 28, 215

Dove, J. R., Shull, J. M., \& Ferrara, A. 2000, ApJ, 531, 846

Gradshteyn, I. S., \& Ryzhik, I. M. 2000, Table of Integrals, Series, and Products, 7th edition, ed. A. Jeffrey, \& D. Zwillinger (Academic Press, Elsevier)

Gulliford, P. 1974, Ap\&SS, 31, 241

Heiles, C. 1979, ApJ, 229, 533

Hnatyk, B., \& Petruk, O. 1999, A\&A, 344, 295

Icke, V. 1988, A\&A, 202, 177

Kompaneets, A. S. 1960, Dokl. Akad. Nauk. SSSR, 130, 1001 [Soviet Phys. Dokl, 5, $46(1960)]$

Kontorovich, V. M., \& Pimenov, S. F. 1998 [arXiv:astro-ph/9802149v1]

Koo, B. C., \& McKee, C. F. 1990, ApJ, 354, 513

Korycansky, D. G. 1992, ApJ, 398, 184

Laumbach, D. D., \& Probstein, R. F. 1969, J. Fluid Mech, 35, 53

Lozinskaya, T. 1992, Supernovae and Stellar Winds in the Interstellar Medium (New York: AIP)

Maciejewski, W., \& Cox, D. P. 1999, ApJ, 511, 792

Mac Low, M.-M., \& McCray, R. 1988, ApJ, 324, 776

Mac Low, M.-M., McCray, R., \& Norman, M. L. 1989, ApJ, 337, 141

Marshall, D. J., Robin, A. C., Reylé, C., Schultheis, M., \& Picaud, S. 2006, A\&A, 453, 635

Newman, W. I., Symbalisty, E. M. D., Ahrens, T. J., \& Jones, E. M. 1999, Icarus, 138,224

Olano, C. A. 1982, A\&A, 112,195

Olano, C. A. 2004, A\&A, 423, 895

Olano, C. A. 2008, A\&A, 485, 457

Rohlfs, K. 1977, Lectures on Density Wave Theory, Lecture Notes in Physics (Springer-Verlag), 69

Rosado, M. 1981, ApJ, 250, 222

Schiano, A. V. R. 1985, ApJ, 299, 24

Sedov, L. I. 1959, Similarity and Dimensional Methods in Mechanics (New York: Academic)

Shapiro, P. R. 1979, ApJ, 233, 831

Silich, S. A. 1992, Ap\&SS, 195, 317

Spitoni, E., Recchi, S., \& Matteucci, F. 2008, A\&A, 484, 743

Spitzer, L. Jr. 1942, ApJ, 95, 329

Tenorio-Tagle, G. 1980, A\&A, 86, 61

Tenorio-Tagle, G. 1981, A\&A, 94, 338

Zel'dovich, Ya. B., \& Raizer, Yu. P. 1968, Physics of Shock Waves and HighTemperature Hydrodynamic Phenomena (New York: Academic) 